ECONomía. Teoría y Práctica • Nueva Época, número 46, enero-junio 2017, pp. 05-40, http://dx.doi.org/10.24275/ETYPUAM/NE/462017/Calderon

\title{
Sincronización comercial e industrial en el TLCAN, un estudio de sincronización de fase
}

\section{Commercial and industrial synchronization in NAFTA, a study on phase synchronization}

\author{
Cuauhtémoc Calderón Villarreal** \\ Reyna Susana García Ruiz, ${ }^{* * *}$ Salvador Cruz Aké****
}

\begin{abstract}
RESUMEN
En este trabajo se contrasta la hipótesis de que los ciclos de crecimiento individuales de los tres países que integran el área del Tratado de Libre Comercio de América del Norte (TLCAN) durante el periodo 1993-2015 han tendido a sincronizarse, para lo cual utilizamos la técnica de sincronización de fase. Ésta permite establecer la causalidad entre variables económicas sin necesidad de hacer los supuestos de esfericidad o ergodicidad tradicionales en el modelado econométrico. Este trabajo aporta evidencia empírica para establecer que los ciclos económicos individuales de Canadá, México y Estados Unidos están sincronizados con las fluctuaciones industriales de Estados Unidos, siendo éste el que encabeza los cambios en las fluctuaciones del área del TLCAN. Adicionalmente, se hacen mediciones sobre la duración de los enganches de sincronización de fase al interior de cada ciclo individual y en el conjunto de los tres ciclos al interior del TLCAN, así mismo se relacionan los periodos de enganche (y su pérdida) de los ciclos con el desempeño macroeconómico de las tres economías estudiadas.
\end{abstract}

Palabras clave: Juglar, ciclo de negocios, integración económica.

Clasificación JEL: E11, E32, F15

\begin{abstract}
In this paper, we contrast the hypothesis that individual growth cycles of the three countries that form the area of the North America Free Trade Agreement (NAFTA) in the period 1993 - 2015 have tended to be synchronized, for which we use the technique of phase synchronization. This allows us to establish causality between economic variables without making assumptions of sphericity or ergodicity, traditional on econometric modeling. This paper provides empirical evidence to confirm that individual business cycles in Canada, Mexico, and the United States are synchronized with industrial fluctuations in the United States, which act as triggers of change for fluctuations in the NAFTA area. In addition, measurements of the duration of the linkage of the phase synchronization within each individual cycle and in all three cycles within NAFTA are made, likewise hitch (and loss) periods are linked with the macroeconomic performance of the three economies studied.
\end{abstract}

Keywords: Juglar, Bussiness Cycle, Economic Integration.

JEL Classification: E11, E32, F15

${ }^{*}$ Fecha de recepción: 11/09/2016. Fecha de aprobación: 24/11/2016.

** El Colegio de la Frontera Norte. Correo: ccalderon22@hotmail.com. ORCID: 0000-00019663-7759

*** Universidad Nacional Autónoma de México. Correo: grreinas@yahoo.com.mx. ORCID: 0000-0001-8677-0169

**** Escuela Superior de Economía. Correo: salvador.ake22@gmail.com. ORCID: 0000-0003$1452-377 \mathrm{X}$ 


\section{INTRODUCCIÓN}

A raíz de la firma del Tratado de Libre Comercio de América del Norte (TLCAN) el $1^{\circ}$ de enero de 1994 se inició un proceso subcontinental de integración económica de los mercados de los tres países de Norteamérica: Estados Unidos, Canadá y México. El objetivo planteado en el artículo 102 del TLCAN fue eliminar obstáculos de comercialización y circulación de mercancías, bienes y capitales, así como la creación de una zona de libre comercio. Los argumentos planteados sustentaban que la especialización en la producción de bienes en los que se tiene una ventaja comparativa y el libre comercio entre los tres países permitirían al consumidor de Norteamérica beneficiarse de los precios bajos en ciertos productos en el extranjero y pagaría por éstos con el ingreso recibido por las exportaciones regionales hechas en bienes en los cuales se mantiene una ventaja comparativa. ${ }^{1}$

De modo que este proceso de integración económica entre los tres países ha propiciado un proceso gradual de integración polarizada y desigual tanto de los mercados como de los respectivos ciclos económicos individuales; donde el ciclo de la economía estadounidense se ha convertido en el polo dominante y los de Canadá y México en periféricos y subordinados (o esclavos). En este artículo se analiza el proceso de sincronización y encadenamiento de los ciclos económicos de los tres países, por lo que se realizará un estudio del orden o jerarquía en la cual los ciclos individuales se encadenan, el tiempo que dura este encadenamiento (o enganche) y el tiempo que duran desenganchados. Las principales aportaciones de este artículo permiten dilucidar el orden y jerarquía en que las variables económicas, determinantes de los ciclos individuales, van presentando cambios y por tanto nos permite definir su causalidad.

Esta clase de estudios económicos, en los cuales se reconoce la complejidad ${ }^{2}$ de los ciclos económicos, responden parcialmente a las críticas de las escuelas heterodoxas del pensamiento económico mediante análisis computacionales del comportamiento fenomenológico de la economía. En particular, el enfoque de sincronización de fase usado en este trabajo incorpora elementos de análisis dinámico al estudio de una problemática inicialmente planteada como un problema estático de un solo equilibrio, lo que enriquece su análisis intertemporal y el establecimiento de relaciones causa-efecto. Además, la flexibilidad de este enfoque

\footnotetext{
${ }^{1}$ Este argumento de ventaja comparativa fue originalmente postulado por Ricardo (1817) y ha sido refutado por Brander y Spencer (1985) (Hollander, 1979) o (Sraffa, 1930), entre otros, aunque la discusión académica continúa.

${ }^{2}$ Se entiende por complejidad económica el hecho de que el sistema económico nunca está en equilibrio, pues las intrincadas relaciones entre los agentes económicos crean constantes ajustes al sistema que, por mucho, superan los efectos de primer orden tradicionalmente analizados.
} 
admite la posibilidad de que los ciclos se enganchen sólo por periodos y que estos "equilibrios de corto plazo" se pueden perder debido a perturbaciones ajenas al sistema económico. Para profundizar sobre el enfoque de complejidad en el estudio de la economía, véase Arthur (2013) o Holling (2001).

Una vez analizada la teoría económica relacionada con ambas hipótesis, se presenta una explicación sobre la forma en que la sincronización de fase permite conocer el tiempo que tardan las variables en sincronizarse y la duración de su enganche, así como las razones técnicas para establecer la dependencia entre las series esclavas y la maestra al interior de cada uno de los países analizados.

Además de lo anterior, el análisis de sincronización de fase constituye una herramienta nueva para describir la dinámica de un sistema tan complejo que en las últimas décadas ha recurrido al análisis de sistemas puramente fenomenológicos, ejemplos de estos últimos pueden ser vistos en Chian, Rempel y Rogers (2006) o Volos, Kyprianidis y Stouboulos (2012), los cuales constituyen una tecnología emergente en el análisis económico cuya principal virtud consiste en que sus resultados no dependen de los tradicionales supuestos econométricos como normalidad, no correlación entre los errores, ergodicidad, homocedasticidad, entre otros. Lo que la compara favorablemente respecto a las metodologías tradicionales de causalidad, como la de Granger (ejemplos de su uso son los trabajos de Thornton (1996) Jung y Marshall (1985) o Chow (1987) y sus variantes de panel (Tekin R. B., 2012), Chang, Simo-Kengne y Gupta (2013) o de Bootstrap (Kar, Nazlığlu y Ağır (2011), Hsueh, Hu y Tu (2013) o Tekin R. B. (2012)) y a otras herramientas econométricas no lineales de causalidad como cópulas (Smith, Gan y Kohn (2012) o Hafner y Manner (2012) otras que son puntualmente abordadas como los métodos de detección de causalidad en sistemas complejos presentada por Sugihara y colaboradores (2012), Wang y Wu (2012) o Nazlioglu (2011) y comparadas con el análisis de sincronización de fase.

Una vez comparada la efectividad de esta metodología contra otras ya usadas en esta clase de estudios, se presentan los resultados de la investigación en la que se determina cuál de las series es la variable maestra de todo el sistema. En la última sección se presenta un análisis de la evidencia empírica presentada.

El articulo está integrado por tres apartados, en el primero se realiza una exposición de las bases teóricas de las principales teorías heterodoxas del ciclo; en el segundo, se exponen las bases científicas del método de sincronización de fase que aplicamos en el artículo; en el último, se presenta un análisis de la evidencia empírica presentada: se detalla la metodología empírica que se utilizó para establecer las zonas de sincronización entre las principales variables macroeconómicas comerciales e industriales de los países que integran el TLCAN, y además se contrastan 
los resultados obtenidos mediante el análisis de causalidad en el sentido de Granger, y el sistema de Vectores Auto Regresivos (VAR).

\section{LAS TEORÍAS HETERODOXAS DEL CICLO ECONÓMICO ENDÓGENO}

Las principales teorías económicas heterodoxas son aquellas que han estudiado los ciclos económicos desde la perspectiva de la acumulación del capital, la inversión y la demanda efectiva.

Sismondi, en Francia, en su obra Les Nouveaux principes realizó uno de los primeros análisis sobre las crisis económicas industriales que apenas habían hecho su aparición hacia 1821 en Inglaterra. Y es el primero en constar que estas crisis de la era del capitalismo industrial naciente del siglo XIX tenían un carácter cíclico y que se explicaban por la sobreproducción.

Para Sismondi, al igual que Malthus, el consumo no es la consecuencia de la producción y afirmaron que no era suficiente incrementar los medios de cambio en la sociedad para incrementar el consumo, sino que "le signe non équivoque de la prospérité de la société, ce n'était pas la production croissante de richesses, mais la demande croissante de travail, ou l'offre croissante de salaire qui le récompense" (Sismondi, 1827: 274). De modo que para estos autores la sobreproducción y demanda efectiva son la causa de las crisis, derivadas de la contracción de la demanda de trabajo y de la caída de los salarios.

De acuerdo con la literatura clásica sobre los ciclos, es posible encontrar una variedad de ciclos económicos superpuestos, vg. Abramovitz (40 meses), Kitchin (3 a 5 años), Juglar (6 a 10 años), Kuznets (16 a 20 años), Kondriatieff (48 a 60 años). Uno de los principales estudiosos del tema, Schumpeter, desarrolla una tipología de los ciclos: cada ciclo Kondratieff, contiene seis ciclos Juglar, los cuales a su vez contienen tres ciclos de Kitchin. Del mismo modo, un ciclo Kuznets contiene dos o tres ciclos Juglar, lo que implica que cada ciclo Kondratieff contiene tres ciclos Kuznets (Calderón, 2012).

Según la teoría marxista la ley que regula en el largo plazo esta dinámica del proceso de acumulación del capital es la ley de la caída tendencial de la tasa de ganancia, por tanto la dinámica de los ciclos económicos está regida por la acumulación del capital. Así, la dinámica de los ciclos está regida por la acumulación del capital. Según Marx (1968), los ciclos económicos modernos son el resultado de una sucesión de estados del sistema capitalista de producción y hacen referencia a la dinámica y reproducción del capital social o global. ${ }^{3}$

\footnotetext{
${ }^{3}$ Para Marx las economías capitalistas siguen una dinámica análoga a "los cuerpos celestes [que] una vez lanzados a sus órbitas las siguen por un tiempo indefinido, lo mismo pasa con la producción social que cuando es lanzada en este movimiento alternativo de expansión y de con-
} 
Desde una perspectiva diferente, para Schumpeter (1935) la dinámica de largo plazo del capitalismo es no lineal (fluctuante), con crisis que marcan la ruptura entre sus fases; y está determinada por el proceso de innovación tecnológico del sistema.

Por su parte, para Keynes (2005) los ciclos económicos son el resultado de las fluctuaciones de la eficacia marginal del capital. Por tanto, asume que los ciclos tendrán una sucesión regular, aunque son modificadas por variaciones de otras variables dentro del sistema económico.

Desde la perspectiva de Kalecki (1977), la dinámica del ciclo económico está determinada por la decisión de invertir el capital fijo privado. De acuerdo con Kalecki (1977), el proceso económico dinámico que encierra las fluctuaciones cíclicas de la economía se explican por el comportamiento de la inversión, ya que la inversión determina el nivel de actividad económica, y el nivel de la actividad económica y su tasa de variación determinan, después de un cierto intervalo de tiempo, a la propia inversión.

\section{SinCRONIZACIÓN DE FASE}

El término sincronización en sistemas complejos se utiliza para determinar si dos sistemas que en forma independiente presentan comportamiento aparentemente caótico o estocástico, al relacionarse pueden estabilizar su dinámica o alguna relación desprendida de ella. En algunos casos se considera la dinámica completa de las series, en otros, como el de esta investigación, se analizan los periodos que presentan y se calcula el diferencial de fase, la cual es conocida como sincronización de fase. Esta última, fue desarrollada por Christian Huygens en 1665, lo que derivó en la teoría de los osciladores acoplados, la cual ha sido abordado mediante ecuaciones diferenciales y por series de tiempo.

El objetivo de utilizar este tipo de sincronización es aprovechar las tendencias de corto y mediano plazo de las series de tiempo. Esto es posible debido a que la amplitud que presentan las series en cada ciclo (valor máximo del ciclo menos valor mínimo del ciclo) es distinta aunque puede ser regular, por lo que para iniciar el análisis se utiliza la duración de cada periodo de una señal $t_{k+1}-t_{k}$ y se compara con la duración de los periodos de la señal a sincronizar. El siguiente paso es determinar la fase de cada una de las series, la cual se define como la cantidad que incrementa en $2 \pi$ sin un ciclo oscilatorio, proporcional a una fracción del periodo, esto es:

tracción lo repite por necesidad mecánica. Los efectos devienen causas y los incidentes primero irregulares y accidentales afectan cada vez más la periodicidad normal”, Marx (1968: 1149-1150). 


$$
\Phi_{X}(t)=2 \pi \frac{t-t_{k}}{t_{k+1}-t_{k}}+2 \pi k
$$

en donde $k$ se refiere al número de periodo o ciclo en el que está la señal. Cuando se trata de un oscilador periódico, en el que cada ciclo tiene la misma duración, por ejemplo la función $f(x)=\sin (x)$, la fase crece uniformemente en el tiempo, ya que todos los elementos de la función $(t)$ serán constantes, el único que cambiará será el número de periodo $k$. Esto se puede apreciar mejor en la gráfica 1.

Gráfica 1. Representación de la función seno y su fase obtenida
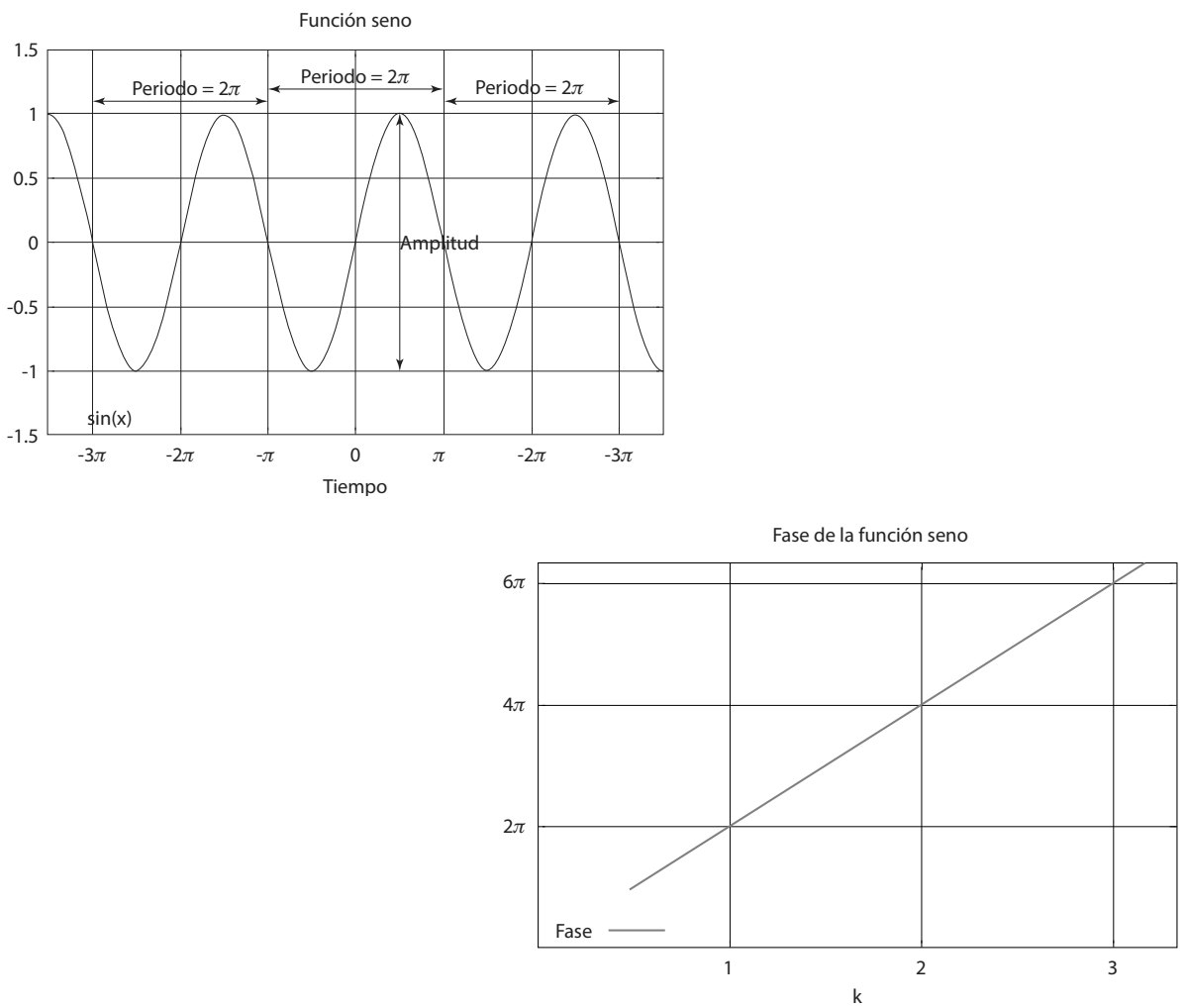

Este procedimiento se realiza para cada una de las variables, aplicando la ecuación (1) se determinan las fases: 


$$
\begin{aligned}
& \Phi_{\text {México }}(t)=2 \pi \frac{t-t_{k}}{t_{k+1}-t_{k}}+2 \pi k \\
& \Phi_{U S A}(t)=2 \pi \frac{t-t_{k}}{t_{k+1}-t_{k}}+2 \pi k \\
& \Phi_{\text {Canadá }}(t)=2 \pi \frac{t-t_{k}}{t_{k+1}-t_{k}}+2 \pi k
\end{aligned}
$$

y después se calcula el diferencial entre éstas. Cuando se mantiene constante en un intervalo de tiempo, se considera que las series están sincronizadas en fase

$$
d \Phi_{X}(t)=\Phi_{\text {México }}(t)-\Phi_{U S A}(t)=c t e
$$

De la diferencia de fase que muestran las series, se obtendrá el sistema maestro, considerándolo como el vector de estado $x_{n}=\left[x_{1}, x_{2}, \ldots, x_{n}\right]$ que es el primero en estabilizar las diferencias y el sistema esclavo $y_{n}$, este es el vector que sigue la dinámica de la variable de salida que es el sistema maestro.

Por lo anterior, el que dos señales estén o no sincronizadas, según Pikovsky y colaboradores (2001), depende de:

- Desajuste de fase. Cuantifica qué tan diferentes son dos señales por medio de un diferencial de fases $d \Phi$, cuando éste es constante se presenta la sincronización.

- Fuerza de acoplamiento. Entre más fuerte sea el acoplamiento entre los sistemas, mayor será la zona de sincronización.

Por medio de esta técnica se puede identificar la sincronía entre periodos y si alguna de ellas muestra algún aceleramiento en sus ciclos, lo cual se realizará en los siguientes apartados. 


\section{SinCRONIZACIÓN DE FASE DE LOS CICLOS DE LOS TRES PAÍSES QUE INTEGRAN AL TLCAN}

\section{III.1. Algunos trabajos empíricos sobre la sincronización de los ciclos}

Antes de presentar los resultados del método de sincronización de fase que aplicamos a los tres países que firmaron el TLCAN, vamos a exponer los resultados de algunos trabajos empíricos previos que fueron realizados solamente sobre la sincronización de los ciclos entre los países Estados Unidos y México básicamente.

Rodríguez Benavides y colaboradores (2015) estudian la sincronización entre los ciclos de Estados Unidos y de México a escala agregada o macroeconómica a partir de la firma del Tratado de Libre Comercio de América del Norte (TLCAN), y para ello utilizan un modelo estructural de series de tiempo múltiple, los resultados de la investigación muestran que a partir de la entrada en vigor del TLCAN no es posible rechazar la hipótesis nula de la existencia de un ciclo económico común entre ambas economías. Sin embargo, la investigación concluye que a pesar de esta evidencia empírica, no está claro que la apertura comercial haya sido el factor explicativo de esta sincronización del ciclo, ya que el comercio bilateral entre ambos países se redujo de manera sustancial en el periodo, sino que dependerá de otros factores endógenos tales como: el gasto público, inversión, empleo, etcétera.

Mejía y colaboradores (2006) analizan, de forma agregada, el proceso de sincronización de los ciclos de Estados Unidos y México siguiendo la clasificación de INEGI (divisiones industriales y ramas de la producción manufacturera). Los autores proponen el uso de coeficientes de correlación para muestras de cinco años. Con su metodología encuentran que a nivel agregado, el grado de asociación de las fluctuaciones industriales y manufactureras de México con el ciclo de Estados Unidos se incrementó sustancialmente tras la firma del TLCAN; segundo, un número importante de ramas productivas siguen al ciclo estadounidense; tercero, las ramas de la división VIII tienen fuertes vínculos con la economía estadounidense.

Delajara (2012), estudia la sincronización entre los ciclos de Estados Unidos y de las regiones de México, este análisis se realiza básicamente con un enfoque regional y suponiendo que existen ciclos regionales en México. Para ello utilizan los índices del Banco de la Reserva Federal de Filadelfia, para medir la actividad económica de Estados Unidos y los índices coincidentes regionales del Banco de México para medir la actividad de las regiones: norte, centro-norte, centro y sur. El autor aplica un modelo estructural lineal de series de tiempo para descomponer los índices coincidentes en tendencia y ciclo y establecer las elasticidades que midan los impactos de los choques provenientes de Estados Unidos sobre las regio- 
nes. Y de acuerdo con los resultados del trabajo existe una variabilidad decreciente de los ciclos regionales del norte al centro ante los choques de Estados Unidos, donde la mayor variabilidad la encontramos en la región norte y la menor en la región centro. Mientras que en el caso de la región sur su variabilidad depende más que nada de los choques específicos de la economía mexicana.

Estas investigaciones cubren la investigación de la sincronización de los ciclos económicos de México y Estados Unidos en tres niveles de abstracción y con métodos empíricos diversos. Rodríguez Benavides y colaboradores (2015) lo abordan desde una perspectiva agregada o macroeconómica donde se constata que existe un alto grado de sincronización a partir de la implantación del TLCAN, pero no se alcanza a explicar solamente por la apertura comercial; Mejía y colaboradores (2006) confirman lo anterior pero analizan también la sincronización a nivel de divisiones industriales y ramas manufactureras concluyendo que la proporción del comercio intra-industrial puede ser un factor explicativo de este alto grado de sincronización de los ciclos de ambos países; y por último, Delajara (2012) realiza un análisis regional y estudia el efecto del ciclo económico estadounidense sobre las fluctuaciones de las regiones de la economía mexicana.

Estas investigaciones tan sólo estudian una parte o dimensión del efecto proceso de integración económica sobre la sincronización de los ciclos económicos; por lo que para entender de manera más clara este proceso de sincronización habría que hacerlo a nivel agregado y a escala subcontinental, tomando en su conjunto a los tres países que firmaron el acuerdo, y así poder analizar de manera más clara y completa las interrelaciones entre los ciclos de los tres países, definiendo así una causalidad clara entre ellos. Es por ello que en el apartado siguiente analizamos la sincronización de fase de los ciclos de los tres países.

\section{III.2. Aplicación del método de sincronización de fase}

El objetivo en este apartado será determinar si existe alguna relación entre las principales variables macroeconómicas comerciales e industriales de los países que integran el TLCAN, por lo que a continuación se explica la metodología empírica que se utilizó para establecer las zonas de sincronización:

1. Se normalizaron las series para los tres países, de tal forma que el máximo de ellas fuera uno, tomando como referencia al valor máximo de cada uno de los indicadores.

2. Se utilizó un filtro para eliminar las perturbaciones de corto plazo y sólo dejar la tendencia del ciclo de cada una de las series de tiempo, en adelante llamado suavizado. 
3. Se observó que las series analizadas presentaban ciclos estocásticos, por lo que se determinó el tiempo de duración de cada ciclo de las señales y se calculó la fase.

4. Se obtuvo el diferencial de las fases de las señales de los distintos países, de tal forma que si eran constantes en un periodo amplio implicaba que se encontraban sincronizadas en fase.

5. Si se presenta sincronización en fase, mostraría la evidencia de que las señales están acopladas al menos en la duración de sus periodos.

Como se había mencionado en el primer apartado, hay una amplia clasificación de la duración de los ciclos en referencia a distintos factores, sin embargo se hará referencia en esta ocasión a los principales movimientos de los factores económicos.

1. Ciclos menores o de corto plazo, que duran en promedio 40 meses. Estos ciclos pueden estar asociados a las variaciones de precios en los alimentos, sugiere que hay una correspondencia entre mínimos y máximos de los precios de los alimentos con los ciclos de corto plazo. También se puede asociar a cambios en los inventarios (duración 3-5 años) según Kitchin (1923).

2. Ciclos principales o de mediano plazo, regularmente compuesto por dos ciclos menores y en menos ocasiones por tres ciclos. Se distingue por una alta tasa bancaria y en algunas ocasiones por pánicos financieros. Dura en promedio 8 años. De tipo Juglar, que fueron estudiados por Marx, Keynes y Kalecki.

3. Movimientos fundamentales o tendencias de 35 años. Depende del comercio internacional o intercambio de dinero con el exterior.

Por lo anterior, se realizará un análisis de corto y mediano plazo, ya que no se cuenta con información suficiente para un análisis de largo plazo. Las series a utilizar son las más representativas de la dinámica macroeconómica comercial e industrial, las cuales van del $1^{\circ}$ de enero de 1993 a febrero de 2015, con datos mensuales, entre los que se encuentran exportaciones, importaciones, consumo privado, actividad industrial manufacturera, etcétera.

A continuación se muestra el resultado del primer paso de la técnica, el cual es dividir cada serie entre su valor máximo histórico, para normalizar las series: 
Gráfica 2. a) Series originales para distintas variables macroeconómicas

b) Series normalizadas para distintas variables macroeconómicas
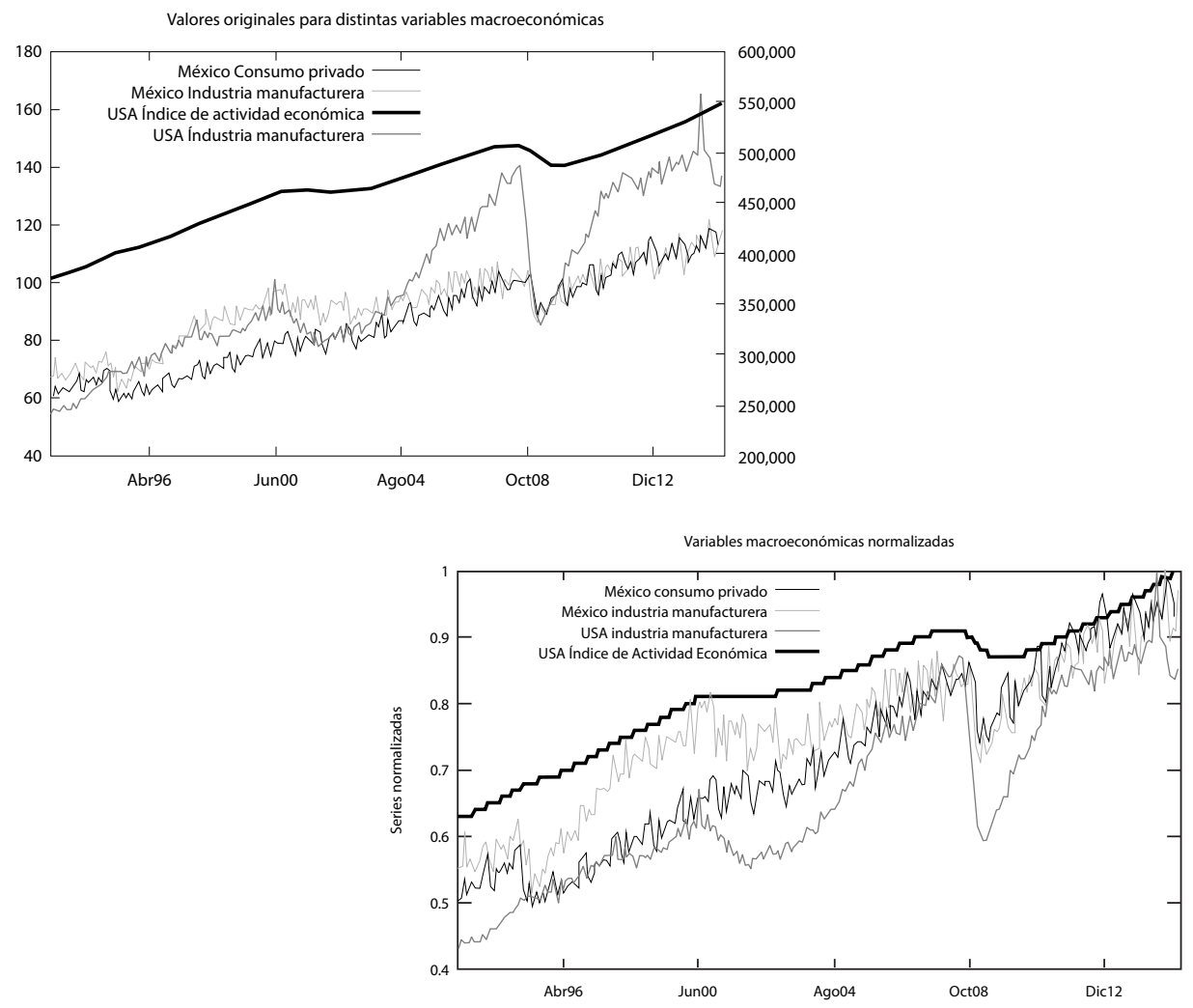

Como se puede observar en la gráfica 2a), se incorporó un segundo eje "y" para poder visualizar las series en una misma gráfica, y en la gráfica 2b) todas las series presentan como valor máximo 1 y no se realizó ninguna modificación en la dinámica, sólo en la escala, por lo que las variables macroeconómicas muestran mayor similitud cuando se normalizan. El siguiente paso es suavizar estas series para quitar las perturbaciones, porque éstas aumentan el número de ciclos de las variables, ya que implican cambios positivos y negativos propios de variaciones de corto plazo pero que no implican un cambio de tendencia, utilizándose la siguiente función:

Serie suavizada $=$ suavizado $^{*}$ Serie suavizada ${ }_{t-1}+(1-\text { suavizado })^{*} \mathrm{x}$ 
en donde $x$ es la variable a suavizar. El suavizado de las series oscila entre valores de (0-1) indicando con valores próximos a cero que se dejarán las tendencias de corto plazo de la serie y con valores próximos a 1 que se quitarán la mayor parte de las oscilaciones, dejando sólo la tendencia.

Como se observa en la gráfica 3 , la serie que capta mejor las variaciones es la que presenta un suavizado de 0.80 , y en la que prevalece sólo la tendencia tiene un suavizado de 0.95 .

\section{Gráfica 3. Distintos suavizados para el consumo privado en México}

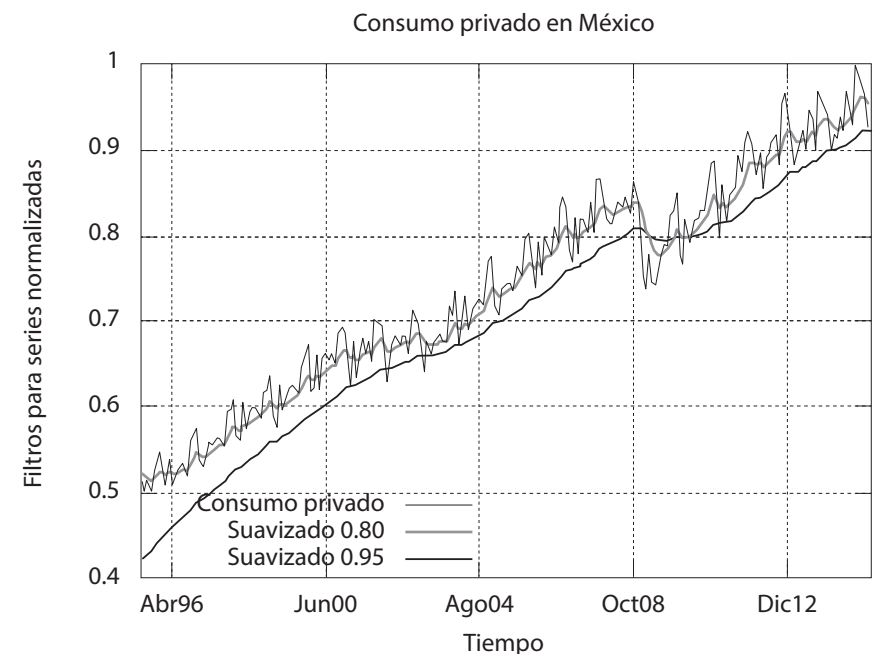

Una vez que se tiene filtrada la serie, se determina el número de ciclos para cada variable, ya que es un elemento fundamental para determinar la fase; en la tabla 2 se presentan los ciclos antes del TLCAN. No se presenta información para variables mexicanas porque no se cuenta con información para los cálculos, sin embargo, se considera importante observar la dinámica de Estados Unidos y Canadá previo al TLCAN. A continuación se muestra un resumen de las variables usadas en el trabajo:

Tabla 1. Nombre de las series y su descripción

\begin{tabular}{ccc}
\hline Serie & País & Descripción \\
\hline SER_M_IM & México & Índice de volumen físico de la industria manufacturera \\
SER_M_CP & México & Índice de volumen físico del consumo privado
\end{tabular}


Tabla 1 (continuación)

\begin{tabular}{|c|c|c|}
\hline Serie & País & Descripción \\
\hline SER_M_Al & México & Índice de volumen físico del total de la actividad industrial \\
\hline SER_M_IFB & México & Índice de volumen físico de la inversión fija bruta \\
\hline SER_US_IM & EUA & Valor de las nuevas órdenes de la industria manufacturera \\
\hline SER_US_OB & EUA & Valor de las nuevas órdenes de bienes duraderos \\
\hline SER_US_M & EUA & Importaciones para consumo final \\
\hline SER_US_CR & EUA & Consumo personal real en bienes duraderos \\
\hline SER_US_IAE & EUA & Índice coincidente de actividad económica \\
\hline SER_C_XAT & Canadá & Exportaciones totales \\
\hline SER_C_XCG & Canadá & Exportaciones de bienes de consumo \\
\hline SER_C_FBCF* & Canadá & Formación bruta de capital fijo (trimestral) \\
\hline
\end{tabular}

Fuentes: Instituto Nacional de Estadística y Geografía (http://www.inegi.org.mx), Saint Louis Federal Reserve (https://fred.stlouisfed.org/) y Statistics Canada, Government of Canada (http://www.statcan.gc.ca/eng/ start).

Tabla 2. Ciclos presentados en las variables macroeconómicas normalizadas previo al TLCAN (01/1988-12/1992)

\begin{tabular}{lc|lc}
\hline Variable & $\begin{array}{c}\text { Número } \\
\text { de ciclos }\end{array}$ & \multicolumn{1}{c}{ Variable } & $\begin{array}{c}\text { Número } \\
\text { de ciclos }\end{array}$ \\
\hline Can Exportaciones totales & 0 & EUA Importaciones & 1 \\
Can Exportación bienes consumo & 0 & EUA Índice de actividad económica & 0 \\
EUA Consumo & 7 & EUA Industria manufacturera & 0 \\
\hline
\end{tabular}

El periodo de análisis es de 6 años, en la industria manufacturera se tiene información a partir de febrero de 1992. Como se puede observar, algunas variables no formaron ningún ciclo, el caso del Índice de la Actividad Económica de Estados Unidos presentó un crecimiento promedio del 0.18 por ciento mensual, no teniendo ningún periodo de decrecimiento, situación similar en otras variables. En el caso del consumo, del mismo país, se presentaron siete ciclos pequeños en 6 años, teniendo una duración promedio de 10.3 meses. 


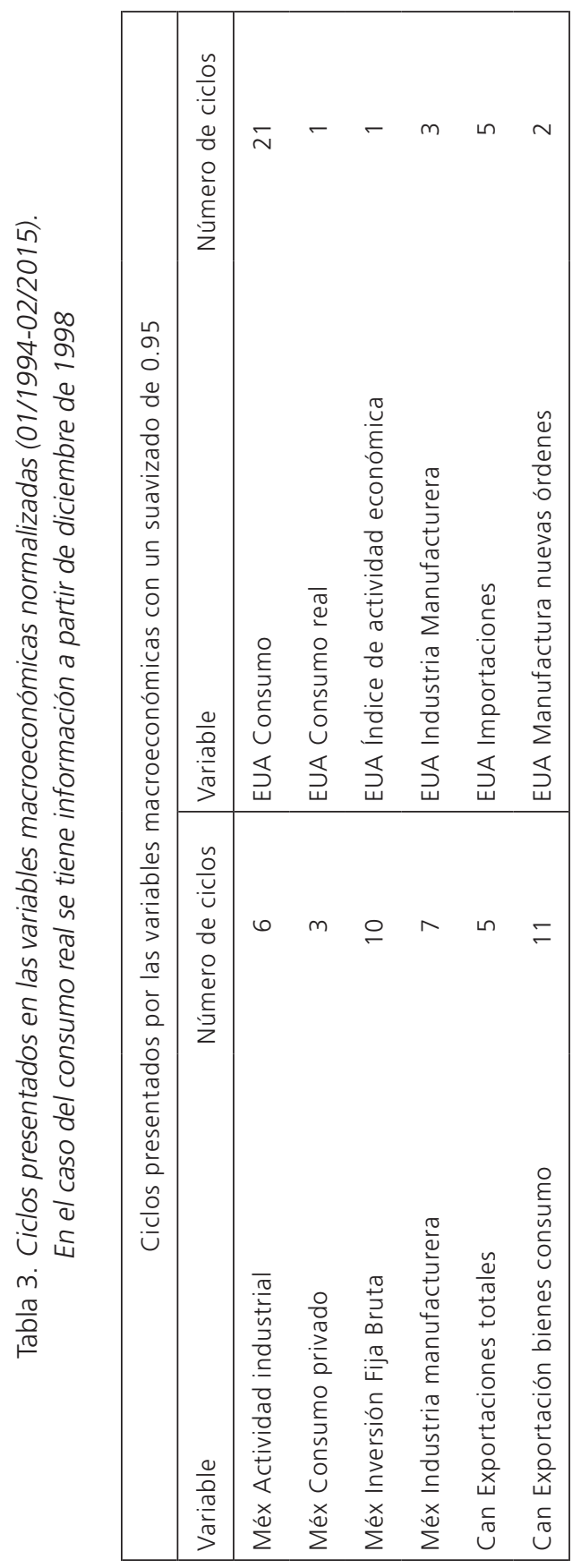




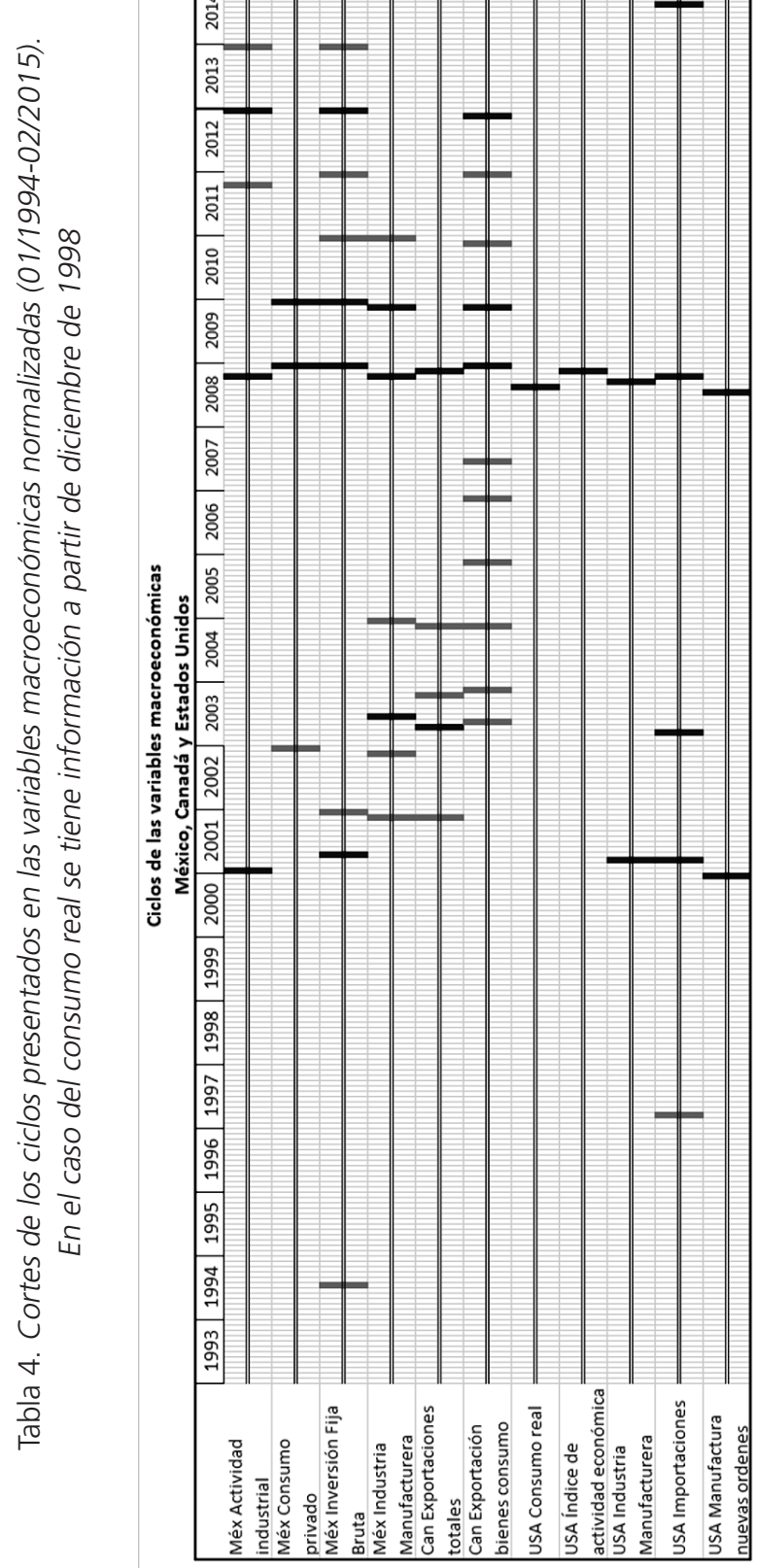


Lo que se puede observar en la tabla 2, para el caso de las exportaciones totales y en bienes de consumo de Canadá, es que en 6 años no se presentó ningún ciclo para ambas variables y después del TLCAN, tabla 3 , se presentaron: cinco ciclos en las exportaciones totales, teniendo un promedio de duración de 4.3 años; y 11 ciclos en la exportación de bienes de consumo, con promedio de duración de 1.9 años.

Respecto a la tabla 4, se pueden observar dos ciclos de mediano plazo (2-3 ciclos cortos de Kitchin). En el primero (enero-febrero de 2001) no todas las variables se sincronizaron debido a la integración paulatina marcada por el TLCAN, sin embargo en el segundo periodo (noviembre-diciembre de 2008) todas las variables macroeconómicas analizadas tienen un corte de ciclo, incluso aquellas como el Índice de Actividad Económica o el Consumo Real, ambas de Estados Unidos, que en 15 años no habían cumplido un ciclo.

Otro aspecto importante que se presenta en la tabla 4, es que en el periodo 2001-2003 las variables macroeconómicas de México y Canadá presentaron un aceleramiento en sus ciclos, que seguramente repercutirá en la sincronización con Estados Unidos.

La variable que se encontró que marcaba el ritmo de la economía mexicana es la Industria Manufacturera de Estados Unidos EUA-IM, ya que de los tres ciclos que presenta en el periodo de enero de 1993 a abril de 2015 siempre se antecedió a los cambios en las variables mexicanas e incluso a las de Canadá. Ejemplo de lo anterior se encuentra en abril de 2001, en el que EUA-IM presentó un corte de ciclo al igual que la Inversión Fija Bruta de México M-IFB, y con un desfase de ocho meses con la Industria Manufacturera de México M-IM y con el mismo tiempo de retardo se cumplió un ciclo con las Exportaciones de Canadá. Aquí todavía no se presentan los mercados totalmente integrados, sin embargo para el primero de octubre de 2008, mes en el que presentó la crisis financiera internacional, la variable EUA-IM estaba presentando un corte de ciclo y sus repercusiones en México y Canadá se presentaron con el siguiente retraso:

1. Con un mes de retraso cumplían sus ciclos las variables:

a) Actividad Industrial de México, M-AI.

b) Industria Manufacturera de México, M-IM.

c) Importaciones de Estados Unidos, EUA-M.

2. Con dos meses de retraso, se presentó para las variables:

a) Exportaciones totales de Canadá, C-X.

b) Índice de Actividad Económica de Estados Unidos, EUA-IAE. No se considera que esta variable presentó sus cambios influenciada por la industria manufacturera, ya que esta variable tiene ciclos de largo plazo. 
3. Con tres meses de retraso, con las variables:

a) Consumo Privado en México, M-CP.

b) Inversión Fija Bruta en México, M-IFB.

c) Exportación en bienes de consumo de Canadá, C-XC.

El tercer ciclo de la industria manufacturera se cumplió en diciembre de 2014, periodo en el que sólo con un mes de retraso tuvieron su cierre de ciclo las variables M-AI y M-IFB. Es importante destacar que aunque las variables M-AI, M-IFB y M-IM son las que se ajustan a los cortes de ciclo de la variable EUA-IM, marcan también el ritmo de cambio en el resto de las variables macroeconómicas de México.

Lo anterior muestra que los tiempos de ajuste entre los ciclos de las variables de Estados Unidos con Canadá y México cada vez van siendo menores. Ya determinados los ciclos de las variables, se calcula la fase para cada uno de los indicadores, planteándose de la siguiente manera:

$$
\begin{aligned}
& \Phi_{\text {Mex_CP }_{-}}(t)=2 \pi \frac{t-t_{k}}{t_{k+1}-t_{k}}+2 \pi k \\
& \vdots \\
& \Phi_{U S A_{-} I M}(t)=2 \pi \frac{t-t_{k}}{t_{k+1}-t_{k}}+2 \pi k
\end{aligned}
$$

\section{Gráfica 4. Diferencias de fase del consumo privado en México} y la industria manufacturera de EUA

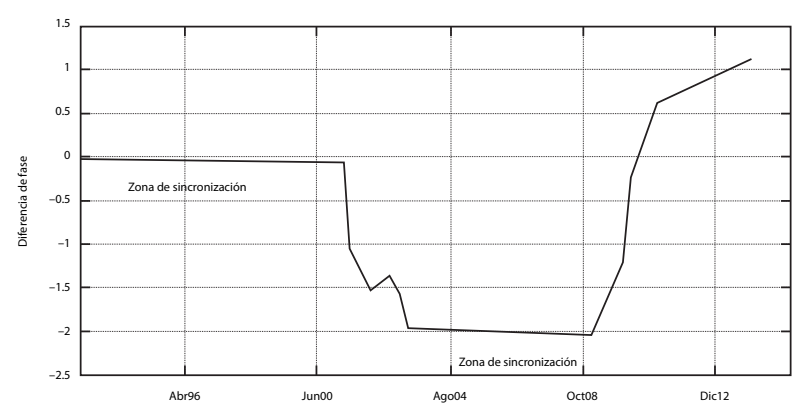

Por lo que se obtiene una nueva serie para cada variable y al calcularse el diferencial de fase se pueden observar los siguientes casos: 
Sincronización de fase, en la cual el diferencial es una constante, como ejemplo se observa al diferencial entre el consumo privado en México y la industria manufacturera en Estados Unidos.

Hasta el periodo de abril de 2001, las fases y por lo tanto los ciclos del consumo privado en México y la industria manufacturera de Estados Unidos estuvieron sincronizados, retomando la sincronía en marzo de 2003 y permaneciendo así hasta diciembre de 2008. Es importante destacar que durante el primer periodo de pérdida de sincronía se presentó la segunda etapa de liberalización de productos agrícolas, mientras que en la segunda pérdida de sincronía se presentó la crisis subprime que inició en octubre de 2008.

Sincronización y pérdida de sincronía. Para el caso de la Inversión Fija Bruta (IFB) de México y las Importaciones M de Estados Unidos se presenta la gráfica 5.

\section{Gráfica 5. Diferencias de fase del consumo privado en México} y la industria manufacturera de EUA

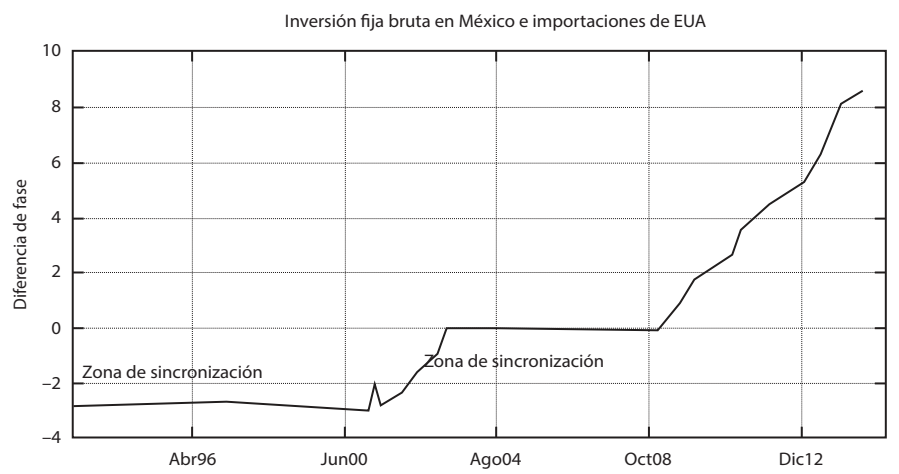

Gráfica 6. Diferencias de fase de la Inversión Fija Bruta de México y las Exportaciones de Canadá

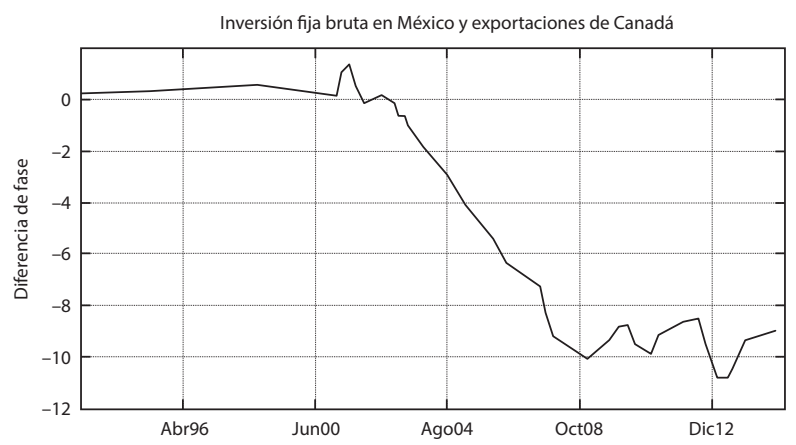


Durante el periodo de enero de 1993 a abril de 2001 estuvieron sincronizadas las variables Inversión Fija Bruta (IFB) de México con las importaciones de Estados Unidos, mostrando un diferencial constante y cumpliendo en ese periodo ambas variables sus ciclos, de abril de 2001 a abril de 2004 se perdió la sincronía debido a que se presentaron dos ciclos de la IFB contra uno de las importaciones de EUA. A partir de diciembre de 2008 se presentaron seis ciclos en LA IFB contra uno de las importaciones, es por ello que no se recuperó la sincronización.

Ausencia de sincronización. Se muestra en la gráfica 6, inversión Fija Bruta respecto a las Exportaciones de Canadá, que hasta abril de 2001 había diferenciales de fase que mostraban que los ciclos estaban débilmente sincronizados; sin embargo, a partir de este periodo los ciclos empezaron a tener diferenciales estocásticos, por lo que no es posible deducir que la dinámica entre México y Canadá este sincronizada. En el siguiente cuadro se muestran los periodos de sincronización de las variables mexicanas respecto a las variables de Canadá y EUA.

En relación con la tabla 5 y la tabla 6 , se puede observar que hay una pérdida de sincronía en el periodo de marzo de 2001 a abril de 2003.

Un aspecto que es importante mencionar es que los periodos de sincronización presentados en la tabla 5 muestran que las diferencias de fase fueron constantes, como el ejemplo de la gráfica 4, sin embargo la gráfica 7 muestra otro tipo de sincronización, en el que los ciclos se aceleran. 
24 ECONOMÍA. TeORÍA Y PrÁCTICA • Nueva Época, número 46, enero-junio 2017

Tabla 5. Sincronización de ciclos presentados en las variables macroeconómicas normalizadas (01/1993-12/2002)

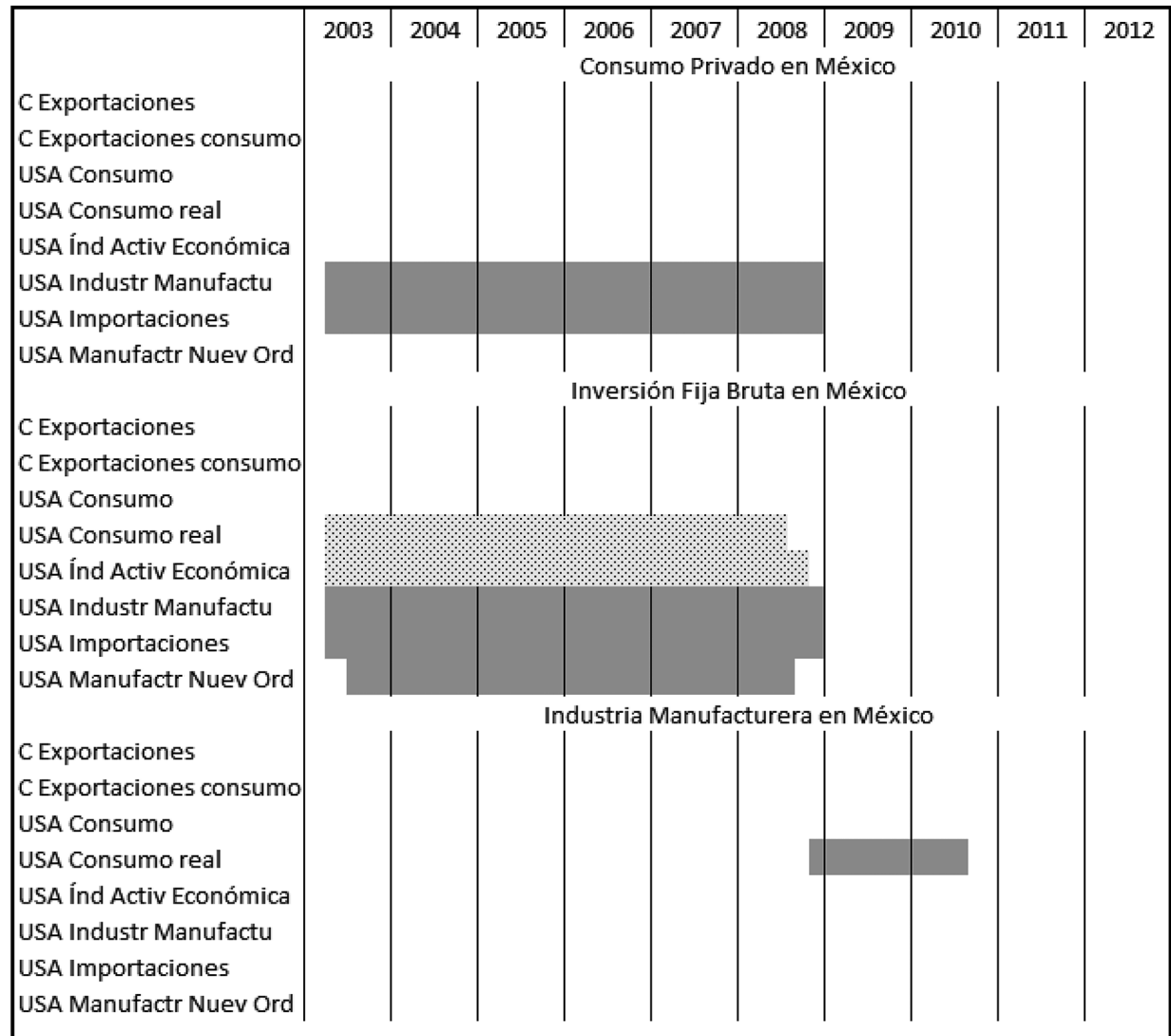

Periodos de sincronización débil

Periodos de sincronización fuerte 
Tabla 6. Ciclos presentados en las variables macroeconómicas normalizadas

(01/2003-12/2012)

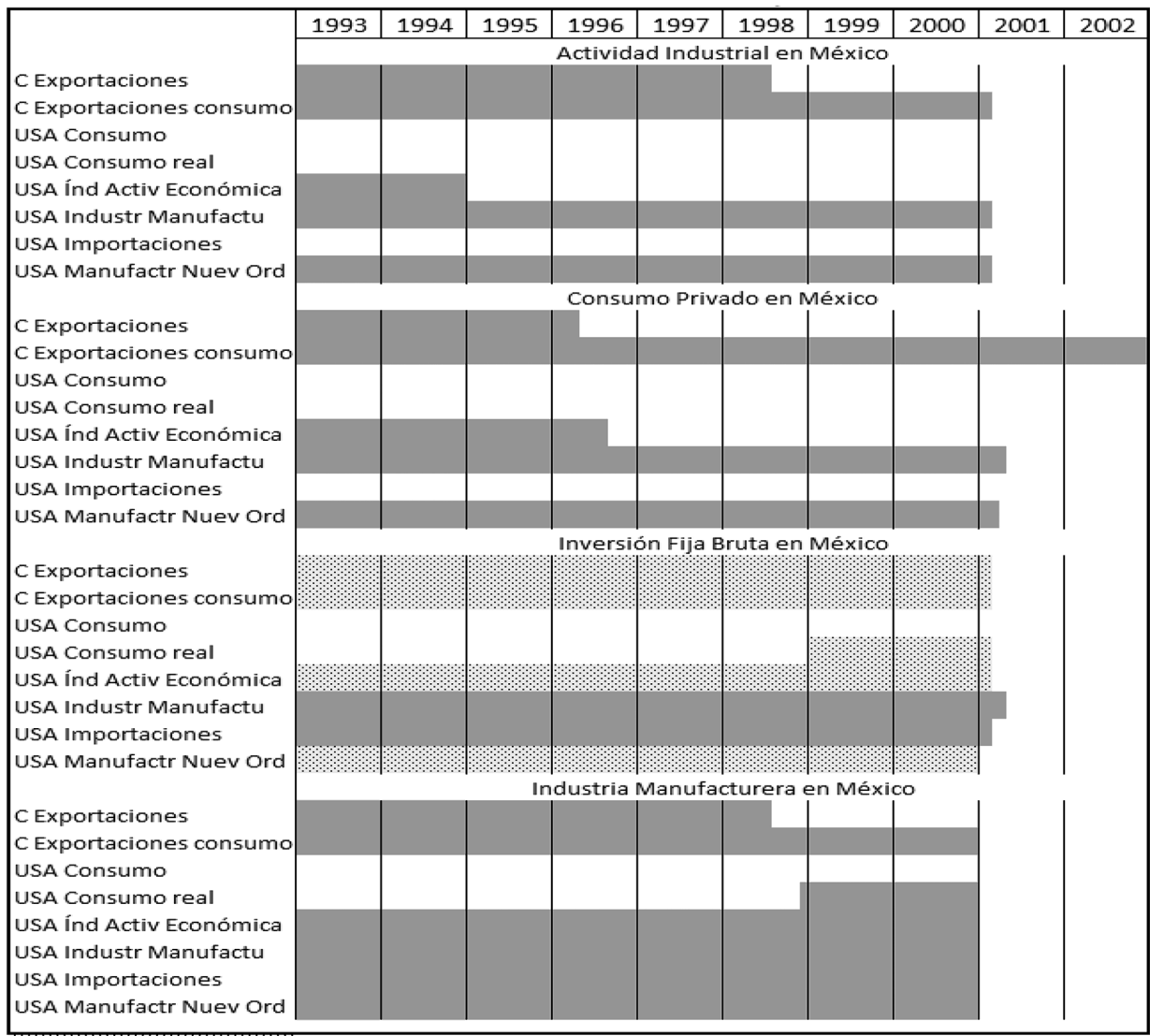

Periodos de sincronización débil

Periodos de sincronización fuerte 
Gráfica 7. Aceleración de los ciclos, detectado por el diferencial de fase: Consumo en México y en Estados Unidos (izquierda); Inversión Fija Bruta en México e Industria Manufacturera en Estados Unidos (derecha)
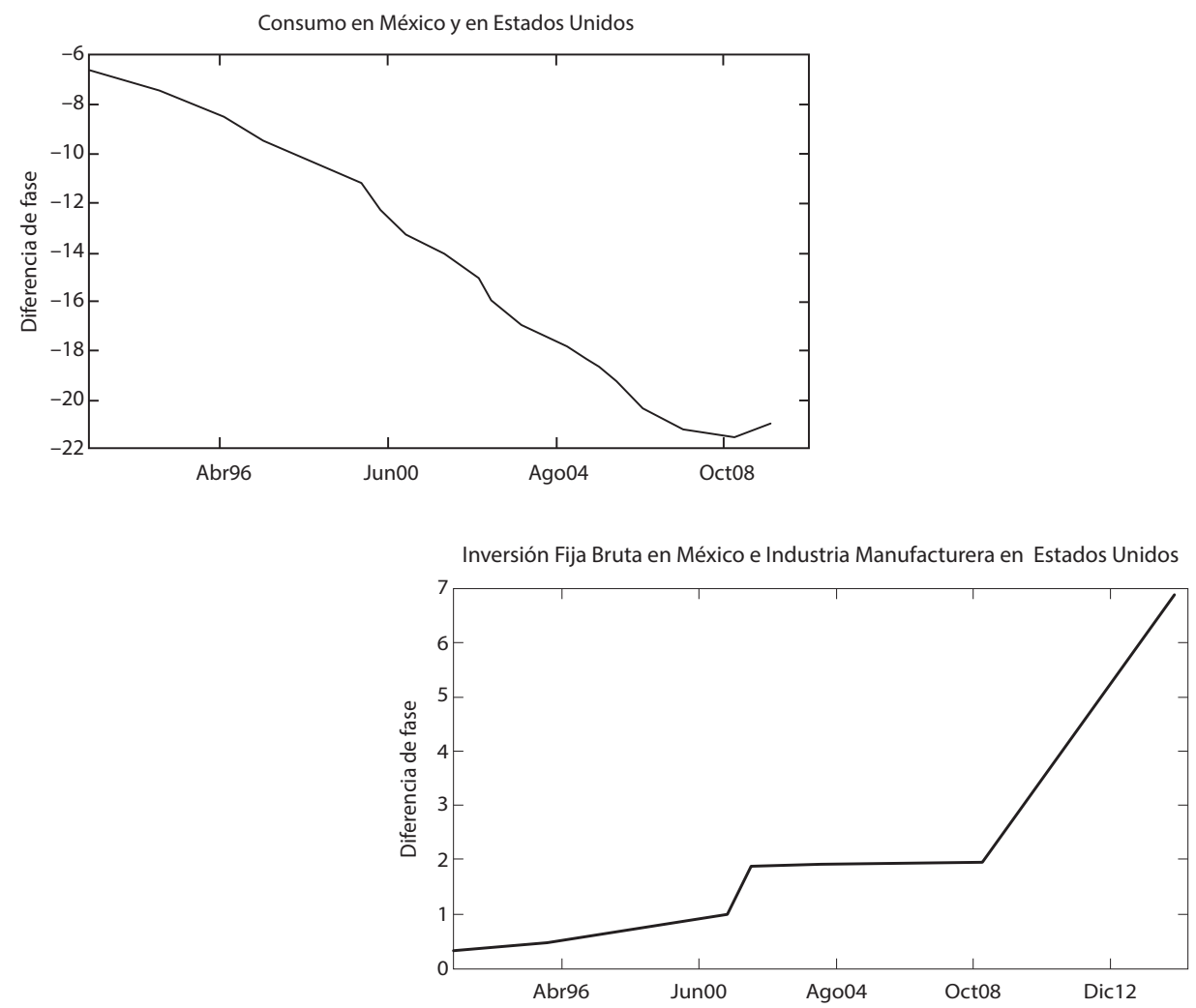

En la gráfica 5 se observa que los ciclos del consumo en Estados Unidos son mucho más rápidos que los ciclos de México, por eso el eje del diferencial de fase muestra valores negativos, caso contrario ocurre con la Inversión Fija Bruta de México y la Industria Manufacturera de Estados Unidos donde durante algunos periodos se aceleraban los ciclos y se presentaban saltos en la serie.

\section{III.3. Otros métodos de detección de dependencia}

Con la finalidad de contrastar los resultados ofrecidos por esta técnica poco utilizada en el análisis económico con otra que puede ser considerada estándar, se presenta un análisis de causalidad basado en el artículo de Toda y Yamamoto 
(1995), la cual adapta la prueba de causalidad de Granger a series cointegradas, corrigiendo el problema que originan tanto el orden de integración de las variables como la naturaleza cointegrante de las series. Es necesario aclarar que, por razones de espacio, no se han incluido las pruebas de estacionariedad KPSS practicadas a cada una de las series, ${ }^{4}$ aunque en todos los casos se tienen variables integradas de orden uno $(m=1)$.

Del mismo modo, es necesario aclarar que para la elaboración del análisis de dependencia de largo plazo (Cointegración), se hizo uso de las series originales (niveles), las cuales son integradas de orden uno. Para estos casos, las series son idénticas a las usadas en el análisis de sincronización y carecen de sufijo.

Tabla 7. Pruebas de rezago óptimo para el modelo vaR

\begin{tabular}{ccccccc}
\hline \multicolumn{7}{c}{ Criterios para la selección del rezago del VAR } \\
\hline \multicolumn{7}{c}{ Variables endógenas: SER_C_XAT SER_C_XCG SER_M_Al SER_M_CP SER_M_IFB } \\
\hline \multicolumn{7}{c}{ SER_M_IM SER_USA_CR SER_USA_IAE SER_USA_IM SER_USA_M SER_USA_OB } \\
\hline Muestra: 1999M01 2015 M02 & Variable exógena: C & Observaciones: 188 \\
\hline Lag & LogL & LR & FPE & AIC & SC & HQ \\
\hline 0 & -10928.55 & NA & $9.66 E+36$ & 116.3782 & 116.5676 & 116.4549 \\
1 & -9058.058 & 3502.198 & $8.00 E+28$ & 97.76658 & 100.039 & 98.68727 \\
2 & -8534.485 & 919.0376 & $1.12 E+27$ & 93.48389 & $97.83931 *$ & $95.24854 *$ \\
3 & -8353.326 & 296.7928 & $6.08 E+26$ & 92.8439 & 99.28235 & 95.45252 \\
4 & -8234.1 & 181.3757 & $6.57 E+26$ & 92.86277 & 101.3843 & 96.31535 \\
5 & -8051.143 & 256.9187 & $3.76 e+26 *$ & $92.2365 *$ & 102.8082 & 96.5002 \\
6 & -7932.935 & $152.1615 *$ & $4.52 E+26$ & 92.23335 & 104.9209 & 97.37386 \\
\hline
\end{tabular}

* Indica el orden del rezago.

Fuente: Elaboración propia con E-Views 9.

Con esta comparación se pretende mostrar que aunque las conclusiones sobre la existencia de dependencia pueden ser similares (tanto en tasas de cambio en el corto plazo como en niveles en el largo plazo), la técnica de sincronización de fase es capaz de marcar los periodos de inicio y término de la sincronización, así como los periodos en que ésta es efectiva, se reduce o en que se pierde, respondiendo a

\footnotetext{
${ }^{4}$ Están disponibles para el lector interesado a vuelta de correo electrónico.
} 
un entorno cambiante (esto es, a procesos estocásticos que pueden no ser constantes a lo largo del tiempo) sin depender de supuestos estadísticos restrictivos. En la tabla 7 se muestran los resultados de las pruebas de rezago para el VAR, esto es el primer paso de la prueba de causalidad planteada por Toda y Yamamoto.

El segundo paso de la metodología es elaborar un VAR que no tenga problemas de correlación serial. Aunque se elaboraron los modelos a 3 y 6 rezagos, éstos resultaron tener mayores correlaciones que el de 5 rezagos, $p=5$ (también plausible, según el resultado presentado en la tabla 7).

Tabla 8. Prueba Lm de correlación de residuales para el modelo var con 5 rezagos

\begin{tabular}{ccc}
\hline \multicolumn{3}{c}{ Prueba LM de correlación de residuales VAR } \\
\hline \multicolumn{3}{c}{ HO: no correlación serial al rezago h } \\
\hline Rezago & \multicolumn{3}{c}{ Muestra: 1999M01 2015M02 } \\
\hline 1 & 145.9679 & 0.0608 \\
2 & 146.3664 & 0.0581 \\
3 & 147.4531 & 0.0513 \\
4 & 147.4158 & 0.0515 \\
5 & 170.56 & 0.002 \\
6 & 149.6341 & 0.0396 \\
\hline
\end{tabular}

Prob chi-cuad con $121 \mathrm{~g}$ de I.

Fuente: Elaboración propia con E-Views9.

En la tabla 8 se muestran los resultados de la prueba LM de correlación serial para el VAR con cinco rezagos, aunque la prueba muestra problemas con el quinto rezago, la prueba es significativa al 1 por ciento para el resto de los rezagos. ${ }^{5} \mathrm{El}$ siguiente paso de la técnica es elaborar un VAR en el cual se agregan el número de rezagos del orden de integración (en este caso, $m+p=6$ ) y se ponen como variables endógenas los rezagos de un orden superior de las variables exógenas $(m+p+1=7)$.

\footnotetext{
${ }^{5}$ Es posible elaborar un modelo con 13 rezagos, aunque presenta problemas en el quinto rezago. Por parsimonia, se elige el modelo de cinco rezagos.
} 
Finalmente ${ }^{6}$ se realiza una prueba de causalidad de Granger, cuyo resumen de resultados se muestran en la tabla 9.

Tabla 9. Resultados de la prueba de causalidad propuesta por Toda y Yamamoto (1995)

\begin{tabular}{c|c|c|c}
\hline \multicolumn{5}{c}{ VAR Causalidad Granger/Prueba de Wald } \\
\hline \multicolumn{5}{c}{ Muestra: 1999M01 2015M02 } \\
\hline Observaciones: 188 \\
\hline Excluidas & Chi-cuad & g de I. & Prob. \\
\hline \multicolumn{5}{c}{ Variable dependiente: SER_USA_CR } \\
\hline Todas & 68.84751 & 50 & 0.0397 \\
\hline Todas & Variable dependiente: SER_USA_IAE \\
\hline & 63.88392 & 50 & 0.0897 \\
\hline Todas & Variable dependiente: SER_USA_M \\
\hline
\end{tabular}

Fuente: Elaboración propia con E-Views9.

Tal y como se puede observar, las pruebas de causalidad propuestas por Toda y Yamamoto (1995) arrojan causalidades similares a las mostradas por el análisis de sincronización, en las que establece que son las series de importaciones de Estados Unidos (SER_EUA_M, su Actividad Económica (SER_EUA_IAE) y posiblemente su consumo real de bienes duraderos (SER_EUA_CR) las que inducen los cambios en el sistema económico asociado al TLCAN. Aunque no establecen si ésta es constante a lo largo de la muestra (en análisis de sincronización sí lo hace). Es importante destacar que la causalidad en el sentido de Granger sólo significa que la varianza explicada de $X$ como función de rezagos de $X$ y $Y$ es mayor que sólo usando rezagos de $X$, mientras que el análisis de sincronización establece cuál es la serie maestra y cuál la seguidora (estableciendo con ello tiempos de reacción entre ambas).

Para el caso del suavizado de largo plazo (.95), y para comparar ambos análisis en niveles, se hace un análisis de cointegración para las variables resultantes del análisis de sincronización de fase, el cual es presentado en la tabla 10.

\footnotetext{
${ }^{6}$ Todos los análisis econométricos, incluyendo los modelos var que se usaron como pasos intermedios para la prueba de Toda-Yamamoto (1995) están disponibles para los lectores interesados a vuelta de correo electrónico.
} 
30 ECONOMÍA. TeORÍA y PRÁCTICA • Nueva Época, número 46, enero-junio 2017

Tabla 10. Resumen de las pruebas de cointegración realizadas al grupo de variables seleccionadas mediante criterios de sincronización

\begin{tabular}{c|c|c}
\hline \multicolumn{2}{c}{ Prueba de cointegración para variables escogidas por criterios de sincronización } \\
\hline $\begin{array}{c}\text { Muestra 1999M01 } \\
\text { 2015M02 }\end{array}$ & Intervalo de rezagos: 1 a 4 & Observaciones: 189 \\
\hline \multicolumn{2}{c}{ Series: SER_C_XAT SER_C_XCG SER_M_AI SER_M_IM SER_EUA_CR SER_EUA_IAE } \\
\hline
\end{tabular}

Número de relaciones cointegrantes por modelo (seleccionados al nivel de.05)

\begin{tabular}{l|c|c|c|c|c}
\hline Tendencia en los datos & Ninguna & Ninguna & Lineal & Lineal & Cuadrática \\
\hline \multirow{2}{*}{ Tipo de prueba } & Sin Intercepto & Intercepto & Intercepto & Intercepto & Intercepto \\
\cline { 2 - 6 } & Sin Tendencia & Sin Tendencia & Sin Tendencia & Tendencia & Tendencia \\
\hline Traza & 3 & 3 & 3 & 3 & 4 \\
\hline Max-Valor propio & 2 & 2 & 2 & 1 & 2 \\
\hline
\end{tabular}

Fuente: Elaboración propia mediante E-Views 9. 


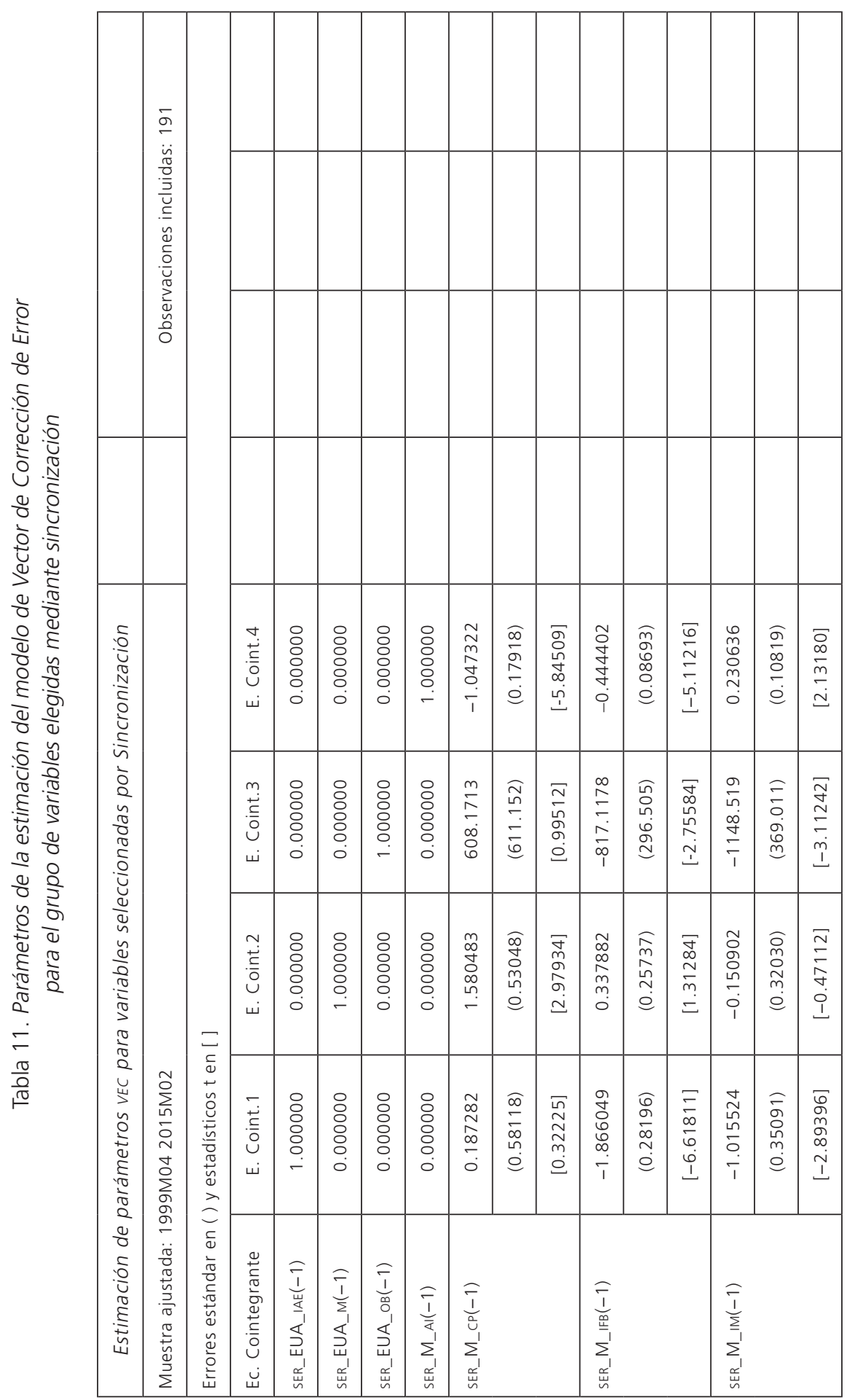




\begin{tabular}{|c|c|c|c|c|c|c|c|c|c|c|c|c|c|c|c|c|c|}
\hline & & & & & 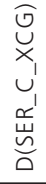 & $\begin{array}{l}\text { o } \\
\text { oे } \\
o \\
\infty \\
\infty \\
\text { i } \\
1\end{array}$ & 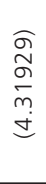 & 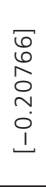 & 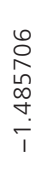 & 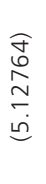 & $\begin{array}{l}\text { J } \\
\text { О } \\
\infty \\
\stackrel{1}{0} \\
0 \\
1\end{array}$ & $\begin{array}{l}\circ \\
\stackrel{0}{\circ} \\
\hat{0} \\
\circ \\
0 \\
0\end{array}$ & 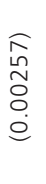 & 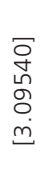 & $\begin{array}{l}\text { o } \\
\hat{o} \\
\infty \\
\infty \\
\vdots \\
\dot{m}\end{array}$ & $\begin{array}{l}\widehat{\bullet} \\
\stackrel{0}{ } \\
\underline{0} \\
\stackrel{0}{=}\end{array}$ & 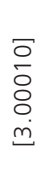 \\
\hline & & & & & 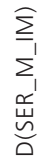 & 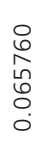 & $\begin{array}{l}\text { a } \\
\text { o } \\
\text { ڤn } \\
\circ \\
\dot{0}\end{array}$ & $\begin{array}{l}\bar{̃} \\
\text { ○े } \\
\stackrel{1}{0} \\
\text { m} \\
=\end{array}$ & 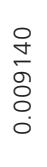 & 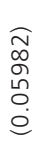 & 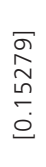 & 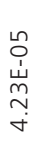 & $\begin{array}{l}\widehat{n} \\
\text { ㅇ } \\
\text { ये } \\
\text { m் }\end{array}$ & 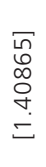 & \begin{tabular}{c}
$\infty$ \\
$\stackrel{\infty}{n}$ \\
\multirow{n}{N}{} \\
m. \\
0
\end{tabular} & 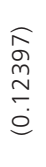 & 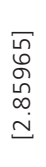 \\
\hline & & & & & 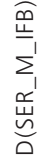 & $\begin{array}{l}\text { } \\
\text { م } \\
\hat{\sigma} \\
\stackrel{0}{0}\end{array}$ & 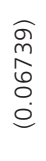 & 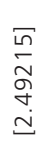 & $\begin{array}{l}\stackrel{8}{\circ} \\
\infty \\
\infty \\
\infty \\
\stackrel{0}{0}\end{array}$ & $\begin{array}{l}\widehat{\circ} \\
\circ \\
\circ \\
\circ \\
\stackrel{0}{0}\end{array}$ & $\begin{array}{l}\overline{0} \\
\bar{\sigma} \\
\dot{0} \\
\dot{m}\end{array}$ & 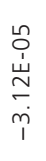 & 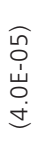 & $\begin{array}{l}\underset{N}{N} \\
\infty \\
\stackrel{1}{N} \\
0 \\
1\end{array}$ & \begin{tabular}{l}
$\infty$ \\
\multirow{J}{0}{} \\
$m$ \\
$m$ \\
$\stackrel{-}{-}$
\end{tabular} & $\begin{array}{l}\widehat{O} \\
\infty \\
\stackrel{\omega}{0} \\
\varrho \\
\stackrel{0}{0}\end{array}$ & \begin{tabular}{l}
$\sqrt[n]{n}$ \\
\multirow{2}{*}{} \\
$\infty$ \\
0 \\
$\dot{0}$
\end{tabular} \\
\hline & & & & & $\begin{array}{l}\widehat{a} \\
\underline{u} \\
\Sigma_{1} \\
\underline{u} \\
\underline{u} \\
0\end{array}$ & $\begin{array}{l}\infty \\
\stackrel{n}{n} \\
\underset{\Sigma}{\sim} \\
\vdots \\
i \\
1\end{array}$ & 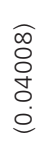 & 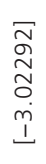 & 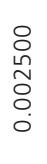 & 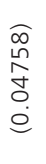 & $\begin{array}{l}\text { J } \\
\stackrel{\text { N }}{N} \\
\text { ก } \\
\dot{0}\end{array}$ & 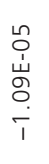 & 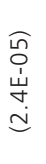 & 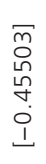 & 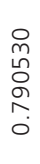 & $\begin{array}{l}\widehat{\overline{0}} \\
\infty \\
\circ \\
\circ \\
0\end{array}$ & $\begin{array}{l}\bar{\sigma} \\
\frac{0}{2} \\
\overline{0} \\
\infty \\
\infty\end{array}$ \\
\hline 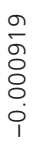 & 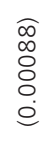 & 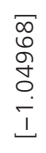 & \begin{tabular}{l}
$\stackrel{n}{\omega}$ \\
$\omega$ \\
$\infty$ \\
$\infty$ \\
\hdashline \\
$\vdots$ \\
0
\end{tabular} & 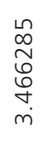 & 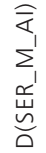 & 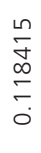 & $\begin{array}{l}\widehat{\sigma} \\
\hat{m} \\
m \\
0 \\
\dot{0}\end{array}$ & 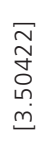 & $\begin{array}{l}\stackrel{n}{\simeq} \\
\underset{\sigma}{N} \\
\frac{0}{0} \\
0\end{array}$ & $\begin{array}{l}\widehat{N} \\
\frac{0}{+} \\
0 \\
\dot{0}\end{array}$ & 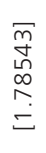 & $\begin{array}{l}\hat{O} \\
\stackrel{1}{u} \\
\ddot{m} \\
\underline{0} \\
\stackrel{-}{-}\end{array}$ & 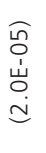 & $\begin{array}{l}F \\
\bar{\Sigma} \\
\infty \\
\circ \\
\stackrel{0}{0} \\
\dot{0}\end{array}$ & 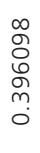 & 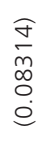 & 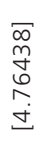 \\
\hline 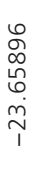 & 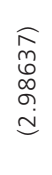 & \begin{tabular}{l}
$\bar{m}$ \\
$\stackrel{N}{N}$ \\
\multirow{N}{*}{} \\
$\stackrel{1}{1}$
\end{tabular} & 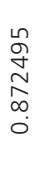 & 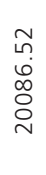 & 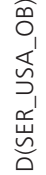 & $\begin{array}{l}\stackrel{+}{m} \\
\stackrel{\infty}{c} \\
\stackrel{m}{m}\end{array}$ & $\begin{array}{l}\widehat{\emptyset} \\
\stackrel{+}{+} \\
0 \\
\dot{\sigma} \\
\bullet\end{array}$ & 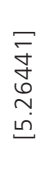 & 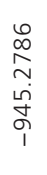 & 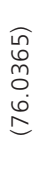 & 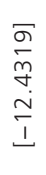 & 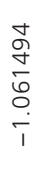 & $\begin{array}{l}\widehat{m} \\
\bar{\infty} \\
m \\
o \\
\dot{0} \\
\dot{0}\end{array}$ & 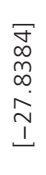 & 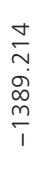 & 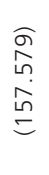 & $\begin{array}{l}\overline{8} \\
8 \\
0 \\
\infty \\
\infty \\
1 \\
1\end{array}$ \\
\hline $\begin{array}{l}\stackrel{\sim}{\Im} \\
\infty \\
\delta \\
\vdots \\
0\end{array}$ & $\begin{array}{l}\widehat{\sigma} \\
\stackrel{1}{ } \\
\stackrel{0}{0} \\
\stackrel{0}{0}\end{array}$ & $\begin{array}{l}\bar{\sigma} \\
\infty \\
\infty \\
\circ \\
\sigma \\
\emptyset\end{array}$ & 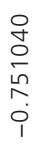 & $\begin{array}{l}\tilde{U} \\
\text { ○ } \\
\stackrel{1}{N} \\
\underset{1}{N}\end{array}$ & 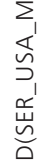 & $\begin{array}{l}\bar{m} \\
6 \\
\tilde{n} \\
0 \\
0 \\
i\end{array}$ & 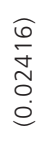 & $\begin{array}{l}\underset{I}{N} \\
\hat{n} \\
\stackrel{i}{i} \\
\stackrel{1}{L}\end{array}$ & $\begin{array}{l}\frac{n}{\kappa} \\
\frac{\sigma}{6} \\
\frac{6}{0} \\
\dot{1} \\
1\end{array}$ & $\begin{array}{l}\widehat{\infty} \\
0 \\
\infty \\
\sim \\
0 \\
\stackrel{0}{0}\end{array}$ & 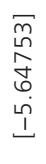 & 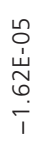 & 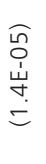 & 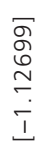 & $\begin{array}{l}0 \\
\infty \\
\infty \\
0 \\
0 \\
0 \\
0 \\
1\end{array}$ & 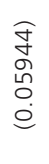 & 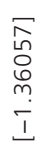 \\
\hline 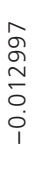 & $\begin{array}{l}\widehat{\Im} \\
\stackrel{0}{ } \\
\stackrel{0}{0} \\
\vdots \\
\dot{0}\end{array}$ & 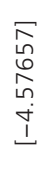 & $\begin{array}{l}\text { ○ } \\
m \\
m \\
\text { o } \\
m \\
\text { ’ }\end{array}$ & 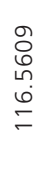 & 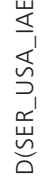 & 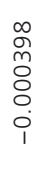 & $\begin{array}{l}\widehat{0} \\
\text { ○े } \\
\circ \\
\circ \\
\dot{0}\end{array}$ & 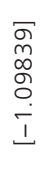 & 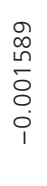 & 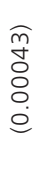 & 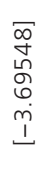 & 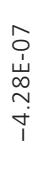 & 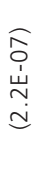 & $\begin{array}{l}\sigma \\
\infty \\
0 \\
\infty \\
o \\
\stackrel{-}{\perp} \\
\end{array}$ & $\begin{array}{l}\frac{n}{N} \\
\frac{0}{8} \\
\circ \\
0 \\
0\end{array}$ & 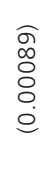 & $\begin{array}{l}\bar{E} \\
\text { ம0 } \\
\text { O } \\
\vdots \\
\dot{0}\end{array}$ \\
\hline 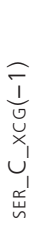 & & & 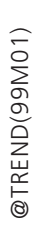 & $\cup$ & 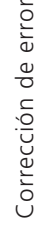 & 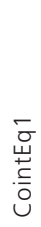 & & & 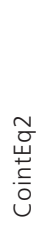 & & & $\begin{array}{l}\stackrel{m}{0} \\
\stackrel{\Psi}{ \pm} \\
\stackrel{\overline{0}}{\cup}\end{array}$ & & & 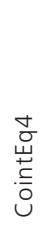 & & \\
\hline
\end{tabular}




\begin{tabular}{|c|c|c|c|c|c|c|c|c|c|c|c|c|c|c|c|c|c|}
\hline 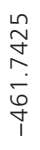 & 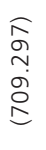 & $\begin{array}{l}\text { न } \\
\circ \\
\circ \\
\hat{n} \\
0 \\
1 \\
1\end{array}$ & $\begin{array}{l}\stackrel{0}{ } \\
\text { Оे } \\
\text { ம} \\
\stackrel{m}{m}\end{array}$ & 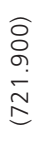 & \begin{tabular}{l}
$\bar{\Sigma}$ \\
oे \\
\multirow{+}{+}{} \\
$\dot{0}$
\end{tabular} & 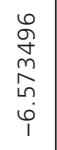 & 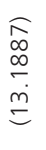 & 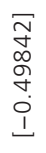 & 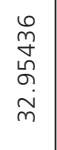 & $\begin{array}{l}\widehat{m} \\
\text { ô } \\
m \\
m \\
\stackrel{=}{=}\end{array}$ & 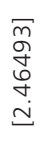 & $\begin{array}{l}\text { ने } \\
\text { ஸू } \\
\circ \\
\circ \\
0 \\
\text { ㅇ }\end{array}$ & $\begin{array}{l}\widehat{O} \\
\stackrel{\leftrightarrow}{1} \\
\stackrel{0}{\circ} \\
0 \\
\stackrel{0}{0}\end{array}$ & 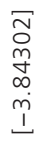 & $\begin{array}{l}0 \\
\sigma \\
\tilde{n} \\
\stackrel{8}{0} \\
\dot{0} \\
1\end{array}$ & 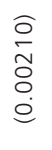 & $\begin{array}{l}\bar{D} \\
\hat{0} \\
\stackrel{0}{0} \\
\infty \\
\dot{-}\end{array}$ \\
\hline 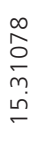 & 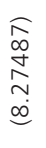 & $\begin{array}{l}\bar{\Xi} \\
\text { ○ } \\
\text { 이 } \\
\infty \\
\vdots \\
\vdots\end{array}$ & $\begin{array}{l}\frac{n}{\sigma} \\
\sigma \\
\frac{6}{6} \\
\frac{\ln }{1}\end{array}$ & 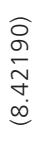 & 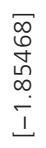 & 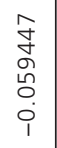 & $\begin{array}{l}\widehat{o} \\
\infty \\
m \\
\stackrel{m}{n} \\
\stackrel{0}{0}\end{array}$ & $\begin{array}{l}\bar{\sigma} \\
\tilde{n} \\
\stackrel{0}{ } \\
m \\
\dddot{0} \\
\stackrel{1}{1}\end{array}$ & $\begin{array}{l}\stackrel{0}{\hat{0}} \\
\frac{\sigma}{m} \\
\frac{m}{0}\end{array}$ & 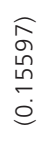 & 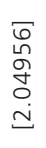 & 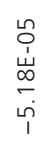 & 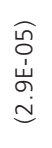 & $\begin{array}{l}\vec{J} \\
\infty \\
\infty \\
\stackrel{1}{N} \\
\stackrel{-}{I}\end{array}$ & 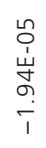 & 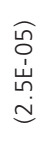 & 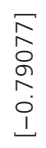 \\
\hline 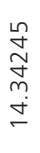 & 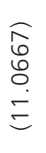 & 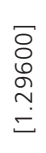 & 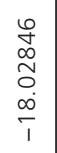 & 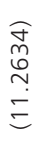 & 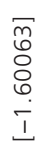 & 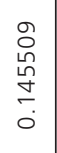 & 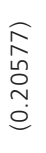 & $\begin{array}{l}\frac{m}{n} \\
\frac{1}{0} \\
\frac{0}{0} \\
0\end{array}$ & 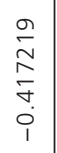 & 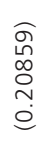 & 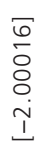 & $\begin{array}{l}\stackrel{0}{0} \\
\dot{1} \\
\dot{\nabla} \\
m \\
m \\
m \\
1\end{array}$ & 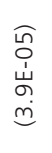 & \begin{tabular}{l}
7 \\
\multirow{N}{N}{} \\
$\infty$ \\
0 \\
0 \\
1 \\
1
\end{tabular} & 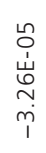 & $\begin{array}{l}\widehat{\text { nn }} \\
\stackrel{1}{ } \\
\dot{m} \\
m \\
m\end{array}$ & $\begin{array}{l}\overline{\hat{0}} \\
\text { o } \\
\sigma \\
0 \\
1\end{array}$ \\
\hline 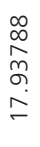 & $\begin{array}{l}\hat{n} \\
\frac{1}{\infty} \\
\infty \\
\\
\dot{e}\end{array}$ & 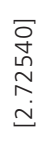 & 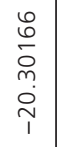 & 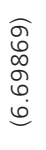 & $\begin{array}{l}\bar{\sigma} \\
\text { } \\
\circ \\
\text { o } \\
\text { m } \\
1\end{array}$ & 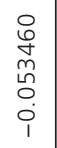 & $\begin{array}{l}\stackrel{\infty}{\infty} \\
\stackrel{N}{N} \\
\stackrel{0}{0}\end{array}$ & \begin{tabular}{l}
$\bar{m}$ \\
$\infty$ \\
0 \\
$m$ \\
\multirow{0}{*}{} \\
0 \\
1
\end{tabular} & $\begin{array}{l}\underset{N}{\infty} \\
\underset{\infty}{\circ} \\
\stackrel{0}{0} \\
\dot{0}\end{array}$ & $\begin{array}{l}\widehat{o} \\
\stackrel{0}{+} \\
\stackrel{ \pm}{ \pm} \\
\stackrel{0}{0}\end{array}$ & $\begin{array}{l}\bar{N} \\
\stackrel{N}{N} \\
\infty \\
0 \\
0\end{array}$ & 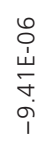 & 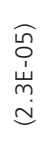 & $\begin{array}{l}\bar{T} \\
\bar{\emptyset} \\
\dot{0} \\
\dot{0} \\
1\end{array}$ & 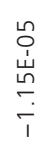 & 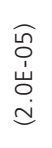 & $\begin{array}{l}\mathbb{1} \\
\infty \\
\infty \\
\infty \\
1 \\
0 \\
1 \\
1\end{array}$ \\
\hline $\begin{array}{l}\bar{\sigma} \\
\sigma \\
0 \\
\sigma \\
6\end{array}$ & 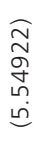 & $\begin{array}{l}\bar{\sigma} \\
\stackrel{8}{\circ} \\
\stackrel{-}{-} \\
\end{array}$ & $\frac{\stackrel{g}{g}}{\stackrel{g}{m}}$ & 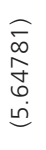 & 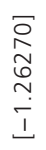 & $\begin{array}{l}\tilde{o} \\
o \\
o \\
m \\
m \\
i \\
1\end{array}$ & $\begin{array}{l}\frac{\infty}{\infty} \\
\hat{0} \\
\frac{0}{0}\end{array}$ & $\begin{array}{l}\bar{\infty} \\
\stackrel{\infty}{m} \\
m \\
m \\
m \\
\stackrel{-}{L}\end{array}$ & $\begin{array}{l}\stackrel{\infty}{N} \\
\stackrel{N}{\tilde{N}} \\
\stackrel{0}{0} \\
\stackrel{0}{0}\end{array}$ & $\begin{array}{l}\widehat{o} \\
\dot{0} \\
\dot{0} \\
\div \\
\stackrel{0}{0}\end{array}$ & $\begin{array}{l}\bar{్} \\
\infty \\
\text { o } \\
\sigma \\
\stackrel{0}{0}\end{array}$ & 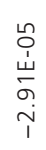 & 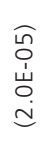 & $\begin{array}{l}\underset{N}{N} \\
\infty \\
+ \\
\stackrel{-}{L}\end{array}$ & $\begin{array}{l}\stackrel{n}{\circ} \\
\stackrel{1}{\sim} \\
\stackrel{N}{N} \\
\frac{1}{1}\end{array}$ & 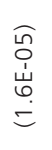 & $\begin{array}{l}\bar{\sigma} \\
\frac{1}{J} \\
0 \\
- \\
1\end{array}$ \\
\hline 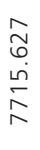 & $\begin{array}{l}\widehat{\partial} \\
\stackrel{\infty}{ } \\
\check{n} \\
\stackrel{0}{\Xi}\end{array}$ & $\begin{array}{l}\overline{\hat{n}} \\
\stackrel{m}{m} \\
\stackrel{n}{\sim} \\
\dot{0}\end{array}$ & \begin{tabular}{l} 
Oे \\
$\infty$ \\
ñ \\
\multirow{1}{*}{} \\
ô \\
1
\end{tabular} & 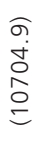 & $\begin{array}{l}m \\
\frac{m}{\infty} \\
\infty \\
0 \\
0 \\
\dot{1} \\
1\end{array}$ & 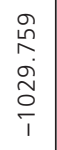 & 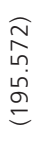 & 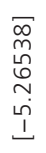 & 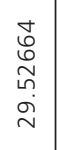 & $\begin{array}{l}\underset{\sigma}{+} \\
\stackrel{\infty}{\infty} \\
\stackrel{\circ}{=}\end{array}$ & $\begin{array}{l}\text { J } \\
\text { D } \\
\stackrel{\infty}{+} \\
\stackrel{0}{\circ}\end{array}$ & $\begin{array}{l}\hat{\omega} \\
\stackrel{0}{0} \\
\text { m } \\
\circ \\
0\end{array}$ & $\begin{array}{l}\text { f } \\
\stackrel{0}{0} \\
\hat{m} \\
0 \\
\stackrel{0}{0}\end{array}$ & $\begin{array}{l}\underset{\sim}{\sim} \\
\underset{\sim}{\infty} \\
\stackrel{0}{0}\end{array}$ & \begin{tabular}{l}
$\infty$ \\
$\stackrel{\infty}{\sim}$ \\
\multirow{\sigma}{*}{} \\
$\dot{0}$ \\
1
\end{tabular} & $\begin{array}{l}\stackrel{\bar{\Xi}}{\sim} \\
\bar{n} \\
\stackrel{0}{0} \\
\dot{0}\end{array}$ & 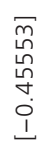 \\
\hline $\begin{array}{l}\text { જે } \\
\infty \\
\infty \\
m \\
\infty \\
\infty \\
0\end{array}$ & $\begin{array}{l}\hat{\omega} \\
\stackrel{n}{\hat{n}} \\
\stackrel{\sigma}{\sigma} \\
\dot{m}\end{array}$ & 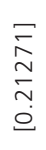 & $\begin{array}{l}\infty \\
\infty \\
\stackrel{N}{N} \\
\infty \\
\infty \\
\end{array}$ & 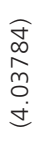 & \begin{tabular}{l}
$\vec{N}$ \\
$\hat{n}$ \\
0 \\
\multirow{J}{*}{} \\
$\dot{1}$ \\
1
\end{tabular} & $\begin{array}{l}\stackrel{\sim}{\sim} \\
\underset{\sigma}{+} \\
\stackrel{\omega}{+} \\
0\end{array}$ & $\begin{array}{l}\underset{\hat{N}}{m} \\
\hat{m} \\
0 \\
\dot{0}\end{array}$ & 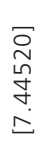 & $\begin{array}{l}\stackrel{0}{ } \\
\sim \\
\infty \\
\stackrel{1}{\sim} \\
\dot{0} \\
i\end{array}$ & \begin{tabular}{l}
$\widehat{\infty}$ \\
\multirow{\sigma}{*}{} \\
$\hat{0}$ \\
$\dot{0}$
\end{tabular} & $\begin{array}{l}\bar{\sigma} \\
\frac{d}{ \pm} \\
\stackrel{1}{1} \\
\text { I }\end{array}$ & 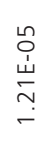 & $\begin{array}{l}\widehat{\widetilde{n}} \\
\stackrel{1}{1} \\
\stackrel{\leftrightarrow}{\forall} \\
\stackrel{=}{=}\end{array}$ & \begin{tabular}{l}
$\bar{\sigma}$ \\
\multirow{J}{*}{} \\
$\infty$ \\
$\stackrel{0}{0}$
\end{tabular} & $\begin{array}{l}\text { 오 } \\
\dot{1} \\
\check{0} \\
\stackrel{-}{-}\end{array}$ & 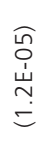 & $\begin{array}{l}\bar{\sigma} \\
m \\
\overline{6} \\
\infty \\
\dot{0}\end{array}$ \\
\hline 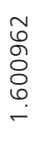 & 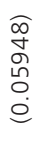 & $\begin{array}{l}\sigma \\
\stackrel{\sigma}{ } \\
\sigma \\
\stackrel{\dot{\omega}}{v} \\
\end{array}$ & 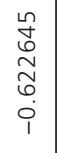 & $\begin{array}{l}\widehat{m} \\
\stackrel{1}{\circ} \\
\stackrel{0}{\circ} \\
0 \\
\stackrel{0}{0}\end{array}$ & $\begin{array}{l}\bar{D} \\
\stackrel{0}{0} \\
\infty \\
\stackrel{1}{1} \\
\stackrel{0}{1} \\
\stackrel{1}{1}\end{array}$ & $\begin{array}{l}\stackrel{N}{n} \\
\stackrel{m}{\circ} \\
\stackrel{\circ}{0} \\
\dot{0}\end{array}$ & $\begin{array}{l}\hat{\Xi} \\
\bar{\sigma} \\
\dot{0} \\
\dot{0}\end{array}$ & 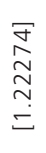 & $\begin{array}{l}\stackrel{D}{N} \\
\stackrel{0}{8} \\
8 \\
0 \\
\dot{0}\end{array}$ & $\begin{array}{l}\widehat{I} \\
\frac{1}{\circ} \\
0 \\
\dot{0}\end{array}$ & \begin{tabular}{l}
$\overline{0}$ \\
\multirow{4}{*}{} \\
\multirow{v}{*}{} \\
6 \\
0 \\
1 \\
1
\end{tabular} & 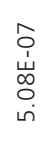 & 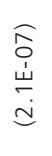 & 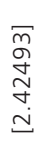 & $\begin{array}{l}\hat{o} \\
\stackrel{1}{1} \\
\text { ه̊ } \\
\stackrel{\sim}{\sim}\end{array}$ & $\begin{array}{l}\widehat{\widehat{O}} \\
\text { 定 } \\
\text { o } \\
=\end{array}$ & 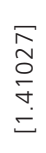 \\
\hline 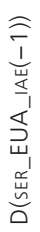 & & & 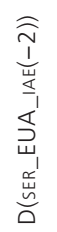 & & & 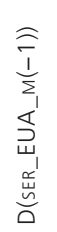 & & & 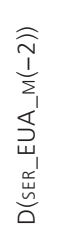 & & & 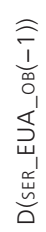 & & & 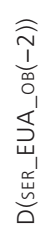 & & \\
\hline
\end{tabular}




\begin{tabular}{|c|c|c|c|c|c|c|c|c|c|c|c|c|c|c|c|c|c|}
\hline $\begin{array}{l}\bar{\sigma} \\
\bar{\sigma} \\
\bar{\sigma} \\
\dot{\infty} \\
m\end{array}$ & 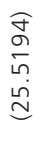 & $\begin{array}{l}\bar{\Xi} \\
\infty \\
\circ \\
\infty \\
+ \\
\Xi \\
=\end{array}$ & 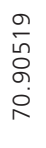 & $\begin{array}{l}\widehat{N} \\
\text { N } \\
\infty \\
\stackrel{0}{ }\end{array}$ & $\begin{array}{l}\bar{N} \\
\text { ڤn } \\
\text { مे } \\
m \\
\text { m }\end{array}$ & 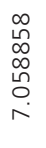 & 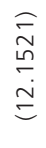 & $\begin{array}{l}\overline{1} \\
\infty \\
0 \\
\infty \\
1 \\
0 \\
0\end{array}$ & 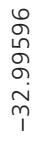 & $\begin{array}{l}\widehat{0} \\
\text { o } \\
\text { ọ } \\
\text { E } \\
=\end{array}$ & 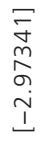 & $\begin{array}{l}\stackrel{0}{m} \\
\stackrel{\leftrightarrow}{m} \\
\stackrel{0}{\circ} \\
\frac{1}{1}\end{array}$ & $\begin{array}{l}\underset{N}{N} \\
\text { En } \\
\stackrel{0}{=}\end{array}$ & 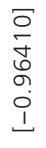 & 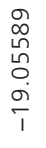 & $\begin{array}{l}\widehat{\infty} \\
\underset{\sim}{U} \\
\mathscr{\sigma} \\
\stackrel{0}{0}\end{array}$ & $\begin{array}{l}\bar{\infty} \\
\infty \\
m \\
\tilde{0} \\
\mathfrak{i} \\
\stackrel{1}{L}\end{array}$ \\
\hline 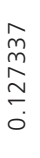 & $\begin{array}{l}\widehat{N} \\
\text { N } \\
\text { } \\
\vdots \\
0\end{array}$ & 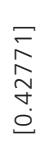 & \begin{tabular}{l}
$\circ$ \\
$\infty$ \\
$\infty$ \\
$\infty$ \\
$m$ \\
\multirow{\sim}{*}{} \\
0
\end{tabular} & 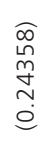 & $\begin{array}{l}\bar{\sigma} \\
\frac{5}{\circ} \\
\infty \\
\vdots \\
=\end{array}$ & \begin{tabular}{l} 
r \\
\multirow{f}{*}{} \\
ô \\
0 \\
0 \\
$\dot{1}$ \\
1
\end{tabular} & $\begin{array}{l}\text { I } \\
\frac{j}{ \pm} \\
\dot{\Xi} \\
\dot{0}\end{array}$ & \begin{tabular}{l}
$\overline{0}$ \\
$\stackrel{1}{*}$ \\
\multirow{8}{*}{} \\
$\dot{1}$ \\
1
\end{tabular} & $\begin{array}{l}\stackrel{0}{+} \\
\frac{6}{0} \\
\tilde{0} \\
0 \\
1\end{array}$ & 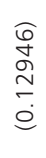 & $\begin{array}{l}\frac{n}{n} \\
\hat{m} \\
\sigma \\
\dot{\sigma} \\
\stackrel{1}{1}\end{array}$ & $\frac{\underset{\infty}{\sigma}}{\underset{ \pm}{ \pm}}$ & $\begin{array}{l}\underset{J}{U} \\
\stackrel{N}{N} \\
\stackrel{0}{0}\end{array}$ & $\begin{array}{l}\overline{\widetilde{\sigma}} \\
\stackrel{0}{\sigma} \\
\sigma \\
\dot{0}\end{array}$ & $\begin{array}{l}\frac{1}{m} \\
\infty \\
\infty \\
\circ \\
0 \\
\dot{0} \\
1\end{array}$ & 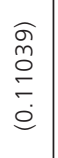 & $\begin{array}{l}\bar{\Xi} \\
\stackrel{0}{ } \\
\infty \\
0 \\
1 \\
1\end{array}$ \\
\hline 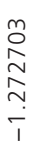 & $\begin{array}{l}\widehat{\sigma} \\
\infty \\
\infty \\
0 \\
0 \\
0\end{array}$ & 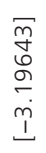 & 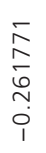 & $\begin{array}{l}\widehat{\hat{N}} \\
\stackrel{0}{n} \\
\stackrel{m}{m} \\
\dot{0}\end{array}$ & $\begin{array}{l}\bar{\sigma} \\
\stackrel{\omega}{n} \\
\stackrel{0}{0} \\
\infty \\
\dot{1} \\
1\end{array}$ & 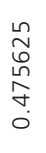 & 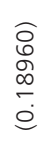 & 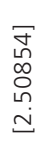 & $\frac{\underset{n}{\stackrel{N}{m}}}{\underset{\sim}{ \pm}}$ & 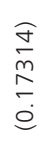 & 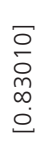 & 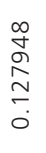 & 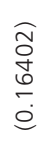 & $\begin{array}{l}\overline{1} \\
\circ \\
\infty \\
\infty \\
\vdots \\
0\end{array}$ & 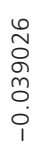 & $\begin{array}{l}\widehat{\tilde{n}} \\
\stackrel{0}{+} \\
\dot{+} \\
\dot{0}\end{array}$ & 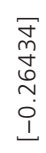 \\
\hline $\begin{array}{l}\hat{\imath} \\
\text { o } \\
0 \\
0 \\
0 \\
0 \\
0 \\
1\end{array}$ & 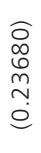 & 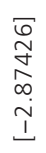 & $\begin{array}{l}\underset{0}{0} \\
\underset{N}{N} \\
\stackrel{0}{0} \\
\dot{1} \\
1\end{array}$ & 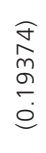 & 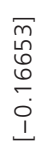 & 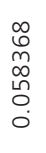 & 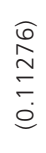 & 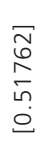 & 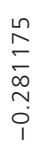 & 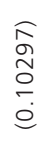 & 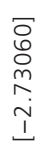 & $\begin{array}{l}\text { N } \\
\text { Nิ } \\
0 \\
0 \\
0\end{array}$ & $\begin{array}{l}\widehat{\Lambda} \\
\text { 年 } \\
\hat{\circ} \\
0 \\
\dot{0}\end{array}$ & $\begin{array}{l}\text { J } \\
\text { o } \\
\text { ปn } \\
\circ \\
\dot{0}\end{array}$ & 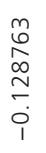 & $\begin{array}{l}\widehat{O} \\
\infty \\
\stackrel{0}{\infty} \\
0 \\
\circ \\
\stackrel{0}{0}\end{array}$ & 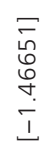 \\
\hline 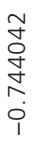 & 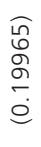 & $\begin{array}{l}\bar{\sigma} \\
\underset{0}{0} \\
\stackrel{N}{N} \\
\dot{m} \\
1\end{array}$ & 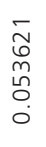 & $\begin{array}{l}\hat{\omega n} \\
m \\
m \\
\hat{\omega} \\
\stackrel{0}{0}\end{array}$ & 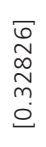 & $\begin{array}{l}\stackrel{ }{N} \\
\underset{0}{\infty} \\
0 \\
O \\
0\end{array}$ & $\begin{array}{l}\widehat{\hat{\sigma}} \\
\text { ํํ } \\
\circ \\
0 \\
\dot{0}\end{array}$ & \begin{tabular}{l}
$\bar{N}$ \\
m \\
\multirow{\infty}{\infty}{} \\
$\dot{0}$ \\
$\dot{0}$
\end{tabular} & \begin{tabular}{l} 
oे \\
寸 \\
D \\
\multirow{2}{*}{} \\
$i$
\end{tabular} & $\begin{array}{l}\widehat{N} \\
\infty \\
0 \\
\infty \\
0 \\
\dot{0}\end{array}$ & $\begin{array}{l}\bar{n} \\
\text { Oे } \\
\stackrel{N}{N} \\
\stackrel{m}{1} \\
1\end{array}$ & 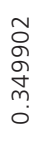 & $\begin{array}{l}\stackrel{1}{N} \\
\underset{\infty}{\infty} \\
0 \\
\dot{0}\end{array}$ & 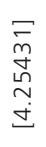 & 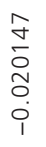 & \begin{tabular}{l}
$\widehat{m}$ \\
$\stackrel{0}{+}$ \\
\multirow{+}{\circ}{} \\
$\dot{0}$
\end{tabular} & $\begin{array}{l}\sqrt[n]{n} \\
\stackrel{N}{N} \\
\vdots \\
0 \\
1\end{array}$ \\
\hline 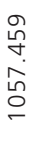 & \begin{tabular}{l}
$\underset{\sim}{\sim}$ \\
\multirow{\sigma}{*}{} \\
$\infty$ \\
$\hat{m}$
\end{tabular} & 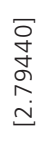 & 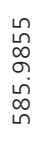 & $\begin{array}{l}\widehat{N} \\
\bar{\sigma} \\
\text { o } \\
\stackrel{m}{m}\end{array}$ & 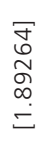 & 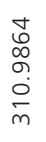 & $\begin{array}{l}\hat{0} \\
\stackrel{0}{1} \\
\stackrel{0}{0} \\
\cong\end{array}$ & 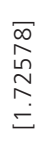 & 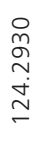 & 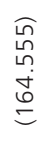 & 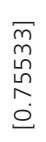 & $\begin{array}{l}\tilde{N} \\
\mathscr{\sigma} \\
\sigma \\
\bar{\omega} \\
m \\
1\end{array}$ & 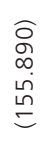 & 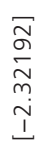 & $\begin{array}{l}\underset{N}{\stackrel{N}{*}} \\
\dot{\omega} \\
\frac{m}{1}\end{array}$ & $\begin{array}{l}\stackrel{f}{ \pm} \\
m \\
o \\
\stackrel{J}{\Xi}\end{array}$ & $\begin{array}{l}\overline{0} \\
0 \\
0 \\
0 \\
0 \\
1\end{array}$ \\
\hline 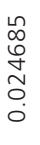 & 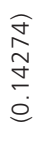 & $\begin{array}{l}\underset{J}{\sigma} \\
\underset{N}{N} \\
\stackrel{0}{0}\end{array}$ & $\begin{array}{l}\stackrel{0}{0} \\
\infty \\
\hat{\sigma} \\
0 \\
\dot{0} \\
1\end{array}$ & $\begin{array}{l}\widehat{\infty} \\
\hat{\sigma} \\
\vdots \\
\dot{0} \\
0\end{array}$ & $\begin{array}{l}\frac{\pi}{2} \\
\frac{1}{\infty} \\
\stackrel{1}{0} \\
0 \\
1\end{array}$ & 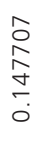 & $\begin{array}{l}\widehat{\hat{\sigma}} \\
\hat{0} \\
0 \\
\dot{0}\end{array}$ & 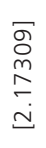 & 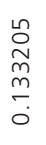 & 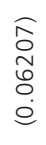 & 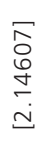 & 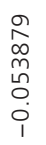 & $\begin{array}{l}\widehat{\circ} \\
\infty \\
\infty \\
ٌ \\
0 \\
\dot{0} \\
\dot{0}\end{array}$ & 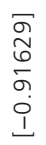 & $\begin{array}{l}\infty \\
\stackrel{\sim}{\sim} \\
\stackrel{0}{0} \\
\dot{0} \\
1\end{array}$ & $\begin{array}{l}\widehat{\text { }} \\
\text { } \\
\text { Nิ } \\
\stackrel{0}{0}\end{array}$ & 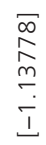 \\
\hline $\begin{array}{l}m \\
\frac{n}{\sigma} \\
\frac{\sigma}{o} \\
o \\
i \\
1\end{array}$ & 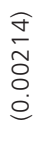 & $\begin{array}{l}\text { J } \\
\text { रे } \\
\text { O } \\
0 \\
0 \\
1\end{array}$ & 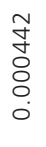 & $\begin{array}{l}\frac{1}{N} \\
\kappa \\
\circ \\
0 \\
\dot{0}\end{array}$ & $\begin{array}{l}\stackrel{\sigma}{\sim} \\
\underset{N}{\tilde{N}} \\
\stackrel{1}{0} \\
\stackrel{0}{0}\end{array}$ & $\begin{array}{l}\frac{N}{m} \\
\frac{N}{0} \\
\stackrel{0}{0} \\
\dot{0}\end{array}$ & $\begin{array}{l}\widehat{ত} \\
\text { O } \\
\circ \\
0 \\
\dot{0}\end{array}$ & 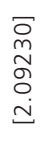 & $\begin{array}{l}\bar{\sigma} \\
\frac{0}{\circ} \\
\circ \\
\circ\end{array}$ & $\begin{array}{l}\widehat{m} \\
\stackrel{0}{\circ} \\
\vdots \\
0 \\
\dot{0}\end{array}$ & $\begin{array}{l}\underset{N}{\hat{N}} \\
\stackrel{0}{\circ} \\
\stackrel{2}{\simeq}\end{array}$ & $\begin{array}{l}\stackrel{0}{1} \\
\frac{0}{\circ} \\
\frac{0}{0} \\
0\end{array}$ & $\begin{array}{l}0 \\
\infty \\
\circ \\
\circ \\
\circ \\
\dot{0}\end{array}$ & $\begin{array}{l}\bar{\sigma} \\
\stackrel{1}{0} \\
\stackrel{0}{0} \\
\stackrel{0}{0}\end{array}$ & 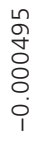 & $\begin{array}{l}\widehat{\sigma} \\
\hat{\circ} \\
\circ \\
\stackrel{0}{0}\end{array}$ & 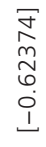 \\
\hline 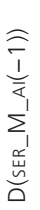 & & & 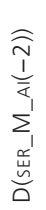 & & & 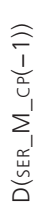 & & & 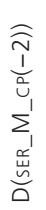 & & & 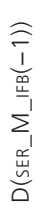 & & & 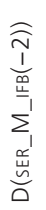 & & \\
\hline
\end{tabular}




\begin{tabular}{|c|c|c|c|c|c|c|c|c|c|c|c|c|c|c|c|c|c|}
\hline $\begin{array}{l}m \\
\infty \\
\stackrel{\infty}{o} \\
\stackrel{0}{0} \\
o \\
o \\
1\end{array}$ & $\begin{array}{l}\text { J } \\
\text { ○ } \\
m \\
m \\
=\end{array}$ & 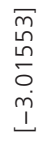 & $\begin{array}{l}\bar{o} \\
\stackrel{0}{0} \\
\underset{1}{N} \\
\underset{1}{1}\end{array}$ & $\begin{array}{l}\underset{ָ}{\infty} \\
m \\
\check{\Xi} \\
\Xi\end{array}$ & 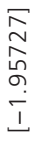 & 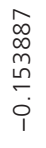 & $\begin{array}{l}\widehat{\infty} \\
\stackrel{0}{0} \\
\sim \\
\stackrel{0}{\circ} \\
\stackrel{0}{0}\end{array}$ & 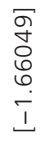 & 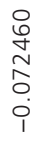 & 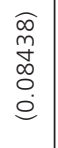 & $\begin{array}{l}\overline{0} \\
\infty \\
\infty \\
\infty \\
\infty \\
0 \\
1 \\
1\end{array}$ & 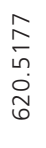 & 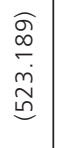 & $\begin{array}{l}\bar{m} \\
\stackrel{0}{0} \\
\infty \\
\vdots \\
=\end{array}$ & 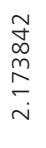 & 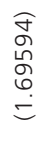 & $\begin{array}{l}\sigma \\
N \\
\infty \\
\\
\vdots\end{array}$ \\
\hline 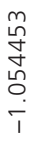 & $\begin{array}{l}\underset{\mathrm{N}}{\mathrm{in}} \\
\stackrel{\mathrm{n}}{0} \\
\stackrel{0}{0}\end{array}$ & 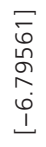 & \begin{tabular}{l}
$\hat{n}$ \\
ô \\
o \\
\multirow{+}{*}{} \\
o. \\
1
\end{tabular} & $\begin{array}{l}\underset{\infty}{\stackrel{\infty}{*}} \\
\underset{m}{\check{D}} \\
\stackrel{0}{0}\end{array}$ & $\begin{array}{l}\frac{\sigma}{\infty} \\
\frac{\infty}{6} \\
\dot{m} \\
1 \\
1\end{array}$ & 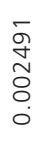 & $\begin{array}{l}\widehat{\infty} \\
\stackrel{0}{\circ} \\
\stackrel{0}{0} \\
\stackrel{0}{0}\end{array}$ & $\begin{array}{l}\overline{\text { nn }} \\
\text { ñ } \\
\text { m } \\
\stackrel{U}{ }\end{array}$ & 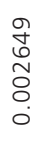 & $\begin{array}{l}\widehat{\infty} \\
\circ \\
\circ \\
\circ \\
\stackrel{0}{0}\end{array}$ & 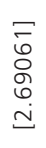 & 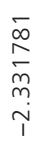 & $\begin{array}{l}\widehat{\infty} \\
0 \\
m \\
0 \\
\stackrel{0}{0}\end{array}$ & 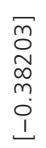 & $\begin{array}{l}\text { O } \\
\stackrel{\leftrightarrow}{n} \\
\stackrel{0}{0} \\
0 \\
0 \\
1\end{array}$ & $\begin{array}{l}\text { o } \\
\text { o } \\
\vdots \\
0 \\
\dot{0}\end{array}$ & $\begin{array}{l}\text { J } \\
\mathbb{0} \\
\stackrel{2}{0} \\
m \\
0 \\
1\end{array}$ \\
\hline 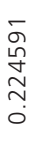 & $\begin{array}{l}\widehat{N} \\
\text { N} \\
\text { O } \\
\text { } \\
0\end{array}$ & $\begin{array}{l}\underset{N}{\sim} \\
\underset{\infty}{0} \\
0 \\
=\end{array}$ & 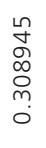 & 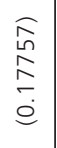 & 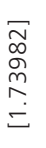 & $\begin{array}{l}\frac{N}{m} \\
\frac{0}{0} \\
0 \\
i\end{array}$ & $\begin{array}{l}\widehat{\text { nn }} \\
\frac{5}{8} \\
\stackrel{0}{0} \\
\stackrel{0}{0}\end{array}$ & $\begin{array}{l}\text { J } \\
\text { ก } \\
\text { ᄋ } \\
\text { o } \\
\text { 1 }\end{array}$ & $\begin{array}{l}\text { N } \\
\stackrel{N}{\cong} \\
0 \\
0 \\
0 \\
i\end{array}$ & $\begin{array}{l}\widehat{\mathbb{v}} \\
\frac{m}{8} \\
\dot{0} \\
\dot{0}\end{array}$ & $\begin{array}{l}\text { I } \\
\text { o } \\
\text { o } \\
\text { I } \\
\text { I }\end{array}$ & 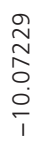 & $\begin{array}{l}\widehat{\partial} \\
\stackrel{0}{0} \\
\hat{\sigma} \\
\stackrel{\infty}{\infty}\end{array}$ & $\begin{array}{l}\bar{\sigma} \\
\infty \\
m \\
m \\
\stackrel{1}{n} \\
\stackrel{-}{I}\end{array}$ & $\begin{array}{l}\text { No } \\
\text { N } \\
\text { m } \\
\stackrel{0}{0} \\
\dot{1}\end{array}$ & 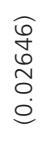 & $\begin{array}{l}\infty \\
\infty \\
o \\
o \\
\tau \\
+ \\
I\end{array}$ \\
\hline $\begin{array}{l}\underset{N}{N} \\
\underset{n}{m} \\
0 \\
1\end{array}$ & $\begin{array}{l}\underset{\mathfrak{N}}{\sim} \\
\stackrel{N}{\sim} \\
\stackrel{0}{0}\end{array}$ & $\begin{array}{l}\bar{I} \\
\text { o } \\
\text { L } \\
\stackrel{1}{1} \\
\text { I }\end{array}$ & 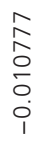 & \begin{tabular}{l}
$\widehat{\bar{\sigma}}$ \\
$\stackrel{2}{\circ}$ \\
\hdashline \\
$\stackrel{0}{0}$
\end{tabular} & $\begin{array}{l}\text { J } \\
\text { 工 } \\
\text { o } \\
\underset{0}{0} \\
\text { I }\end{array}$ & $\begin{array}{l}\stackrel{\infty}{N} \\
\underset{m}{\circ} \\
\stackrel{0}{0} \\
\dot{0}\end{array}$ & $\begin{array}{l}\widehat{0} \\
\infty \\
\circ \\
\circ \\
\stackrel{0}{0}\end{array}$ & 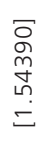 & 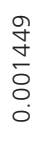 & $\begin{array}{l}\widehat{\infty} \\
\stackrel{0}{\circ} \\
\stackrel{0}{0} \\
\stackrel{0}{0}\end{array}$ & 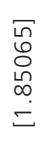 & 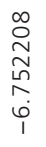 & \begin{tabular}{l}
$\widehat{O}$ \\
$\infty$ \\
\multirow{L}{*}{} \\
$\infty$ \\
$\dot{J}$ \\
$\dot{J}$
\end{tabular} & $\begin{array}{l}\bar{m} \\
\infty \\
\circ \\
\varnothing \\
m \\
\stackrel{-}{\perp} \\
\end{array}$ & 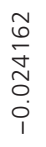 & $\begin{array}{l}\text { } \\
\text { மn } \\
\vdots \\
0 \\
\dot{0}\end{array}$ & 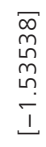 \\
\hline \begin{tabular}{l}
0 \\
0 \\
\multirow{+}{+}{} \\
$\sigma$ \\
\\
0 \\
1
\end{tabular} & $\begin{array}{l}\widehat{0} \\
0 \\
0 \\
0 \\
\dot{0}\end{array}$ & $\begin{array}{l}\bar{T} \\
\infty \\
\curvearrowright \\
\infty \\
\vdots \\
1\end{array}$ & \begin{tabular}{l}
\multirow{N}{*}{} \\
$\dot{0}$ \\
$\stackrel{0}{0}$ \\
0 \\
$\dot{1}$ \\
1
\end{tabular} & $\begin{array}{l}\widehat{夭} \\
\stackrel{\circ}{ } \\
\infty \\
\circ \\
\stackrel{0}{0}\end{array}$ & 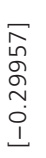 & $\begin{array}{l}\bar{\sigma} \\
\bar{\circ} \\
8 \\
\dot{0}\end{array}$ & $\begin{array}{l}\widehat{m} \\
\hat{\circ} \\
\circ \\
\dot{0} \\
\dot{0}\end{array}$ & $\begin{array}{l}\frac{\pi}{n} \\
\stackrel{n}{n} \\
\dot{0}\end{array}$ & 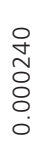 & $\begin{array}{l}\widehat{0} \\
\stackrel{0}{0} \\
\circ \\
\stackrel{0}{0}\end{array}$ & $\begin{array}{l}\mp \\
\underset{n}{n} \\
\text { m } \\
\dot{0}\end{array}$ & $\begin{array}{c}\frac{\sigma}{N} \\
\underset{N}{\infty} \\
\infty \\
\dot{1} \\
1\end{array}$ & $\begin{array}{l}\widehat{\sigma} \\
\frac{1}{m} \\
\stackrel{\circ}{\circ} \\
\dot{ \pm}\end{array}$ & $\begin{array}{l}\bar{D} \\
\dot{J} \\
\dot{b} \\
6 \\
- \\
\end{array}$ & $\begin{array}{l}\underset{N}{N} \\
\underset{N}{N} \\
0 \\
i \\
i\end{array}$ & $\begin{array}{l}\underset{\hat{N}}{\tilde{m}} \\
\stackrel{0}{0} \\
\dot{0}\end{array}$ & 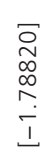 \\
\hline 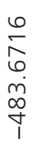 & $\begin{array}{l}\stackrel{\text { }}{N} \\
\text { } \\
\stackrel{\sigma}{=} \\
=\end{array}$ & 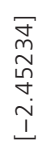 & 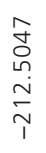 & $\begin{array}{l}\widehat{\infty} \\
0 \\
0 \\
\infty \\
0 \\
\varrho\end{array}$ & $\begin{array}{l}\underset{n}{n} \\
\stackrel{\sigma}{n} \\
\stackrel{2}{\sim} \\
\stackrel{+}{1}\end{array}$ & 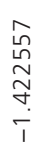 & 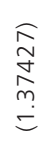 & 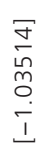 & 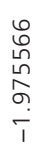 & $\begin{array}{l}\stackrel{\widehat{O}}{\stackrel{n}{n}} \\
\stackrel{n}{\stackrel{n}{n}} \\
\stackrel{=}{=}\end{array}$ & 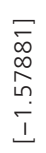 & 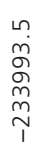 & 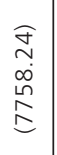 & $\begin{array}{l}\overline{0} \\
\dot{0} \\
\ddot{0} \\
\dot{0} \\
1\end{array}$ & 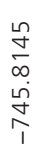 & 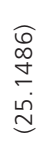 & 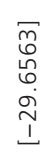 \\
\hline $\begin{array}{l}\bar{\sigma} \\
\infty \\
\infty \\
\infty \\
0 \\
0 \\
i \\
1\end{array}$ & 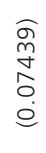 & $\begin{array}{l}\bar{n} \\
\text { மn } \\
\tilde{n} \\
\tilde{1} \\
1\end{array}$ & $\begin{array}{l}\text { O } \\
\infty \\
\stackrel{+}{+} \\
\text { m} \\
0 \\
\dot{1} \\
1\end{array}$ & $\begin{array}{l}\widehat{0} \\
\stackrel{0}{0} \\
\hat{o} \\
\ddot{0}\end{array}$ & $\begin{array}{l}\bar{\pi} \\
\tilde{m} \\
0 \\
\text { in } \\
\dot{0} \\
\dot{1}\end{array}$ & $\begin{array}{l}\circ \\
\frac{0}{\circ} \\
\circ \\
\circ \\
\circ\end{array}$ & 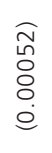 & 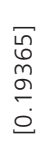 & 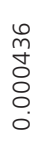 & 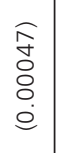 & 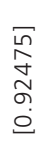 & \begin{tabular}{l}
0 \\
\multirow{U}{0}{} \\
$\stackrel{2}{0}$ \\
$\stackrel{\sim}{1}$ \\
$\sim$
\end{tabular} & 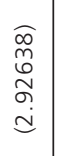 & 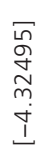 & 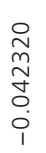 & $\begin{array}{l}\text { क़ } \\
\text { ু } \\
\circ \\
\stackrel{0}{0}\end{array}$ & $\begin{array}{l}\overline{\bar{m}} \\
\bar{b} \\
\dot{g} \\
\dot{I}\end{array}$ \\
\hline 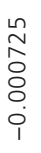 & $\begin{array}{l}\widehat{N} \\
\bar{\sigma} \\
\text { ○ } \\
\dot{0} \\
0\end{array}$ & \begin{tabular}{l}
$\bar{T}$ \\
$\hat{\sigma}$ \\
\multirow{0}{0}{} \\
$\dot{0}$ \\
1 \\
1
\end{tabular} & 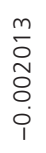 & $\begin{array}{l}\widehat{\pi} \\
\stackrel{2}{\circ} \\
\circ \\
\circ \\
0\end{array}$ & 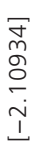 & 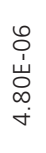 & 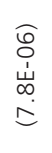 & $\begin{array}{l}\bar{\sigma} \\
\infty \\
\stackrel{0}{\sigma} \\
\dot{0} \\
0\end{array}$ & $\begin{array}{l}\bullet \\
0 \\
\dot{山} \\
\circ \\
\infty \\
\dot{1}\end{array}$ & 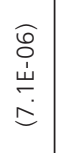 & $\begin{array}{l}\bar{m} \\
\stackrel{n}{\sigma} \\
\sigma \\
\infty \\
\dot{0}\end{array}$ & \begin{tabular}{l}
\multirow{N}{0}{} \\
$\infty$ \\
0 \\
0 \\
$\vdots$ \\
1
\end{tabular} & 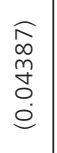 & 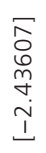 & $\begin{array}{l}0 \\
\frac{0}{m} \\
\circ \\
\circ \\
0 \\
\dot{0} \\
1\end{array}$ & $\begin{array}{l}\text { ণ } \\
\stackrel{\circ}{\circ} \\
\stackrel{0}{0}\end{array}$ & 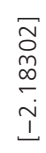 \\
\hline 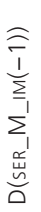 & & & 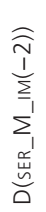 & & & 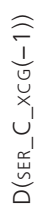 & & & 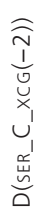 & & & $\cup$ & & & 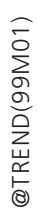 & & \\
\hline
\end{tabular}




\begin{tabular}{|c|c|c|c|c|c|c|c|c|c|c|c|c|c|c|}
\hline $\begin{array}{l}\frac{9}{5} \\
\text { م } \\
\frac{0}{0} \\
0 \\
0 \\
1\end{array}$ & $\begin{array}{l}\widehat{N} \\
\overline{8} \\
\dot{0} \\
\dot{0}\end{array}$ & 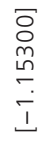 & $\begin{array}{l}\stackrel{+}{N} \\
\stackrel{N}{\infty} \\
\infty \\
\stackrel{0}{0} \\
0\end{array}$ & $\begin{array}{l}\stackrel{\infty}{\stackrel{N}{\sim}} \\
\underset{\sim}{\sim} \\
\stackrel{0}{0} \\
0\end{array}$ & 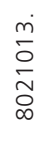 & 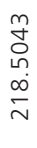 & 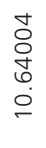 & $\begin{array}{l}\underset{J}{J} \\
\underset{N}{\infty} \\
\stackrel{\infty}{N} \\
\frac{1}{1}\end{array}$ & $\begin{array}{l}\text { O } \\
\stackrel{+}{N} \\
\stackrel{N}{m} \\
\stackrel{m}{\sim}\end{array}$ & 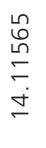 & $\begin{array}{l}\stackrel{\hat{\omega}}{\stackrel{0}{\sigma}} \\
\frac{\sigma}{\sigma} \\
0\end{array}$ & 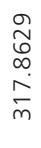 & & \\
\hline $\begin{array}{l}\stackrel{\varphi}{0} \\
\dot{1} \\
\text { 员 } \\
m \\
\infty\end{array}$ & 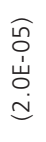 & $\begin{array}{l}\bar{m} \\
\infty \\
0 \\
\dot{y} \\
\dot{0} \\
\dot{0}\end{array}$ & $\begin{array}{l}\frac{\infty}{0} \\
\frac{0}{j} \\
\vdots \\
0\end{array}$ & 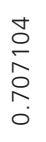 & $\begin{array}{l}\bar{\infty} \\
0 \\
\dot{-} \\
\circ \\
\circ\end{array}$ & $\begin{array}{l}\stackrel{\infty}{m} \\
\stackrel{n}{\tilde{L}} \\
\stackrel{\sim}{\sim}\end{array}$ & 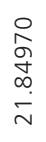 & 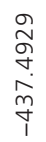 & $\begin{array}{l}\frac{n}{\sigma} \\
\frac{\pi}{\infty} \\
\infty \\
\dot{\sigma}\end{array}$ & 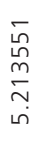 & 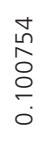 & 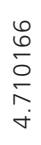 & & \\
\hline 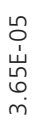 & 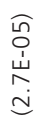 & $\begin{array}{l}\bar{n} \\
6 \\
6 \\
m \\
= \\
=\end{array}$ & 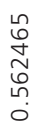 & 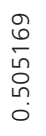 & 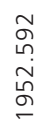 & 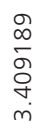 & $\begin{array}{l}n \\
\infty \\
\hat{\sigma} \\
\infty \\
\infty \\
\sigma\end{array}$ & $\begin{array}{l}m \\
\text { O } \\
\text { o } \\
\text { mं } \\
\text { ơ } \\
\text { I }\end{array}$ & 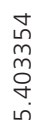 & 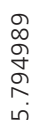 & $\begin{array}{l}\bar{\infty} \\
\stackrel{n}{+} \\
\stackrel{\omega}{n} \\
\dot{0}\end{array}$ & 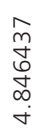 & & \\
\hline $\begin{array}{l}\stackrel{2}{0} \\
\text { 足 } \\
\stackrel{\leftrightarrow}{夭} \\
\stackrel{\sim}{\sim}\end{array}$ & 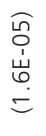 & 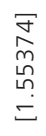 & 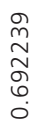 & 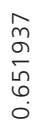 & 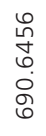 & 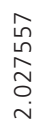 & 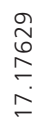 & $\begin{array}{l}n \\
\infty \\
0 \\
N \\
m \\
m \\
m \\
1\end{array}$ & $\begin{array}{l}\infty \\
0 \\
0 \\
\dot{\sigma} \\
\text { m } \\
\forall\end{array}$ & 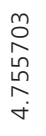 & 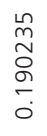 & \begin{tabular}{l}
$\stackrel{ }{N}$ \\
$\hat{v}$ \\
$m$ \\
\multirow{v}{*}{} \\
$\dot{m}$
\end{tabular} & 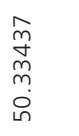 & \\
\hline 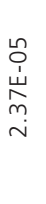 & $\begin{array}{l}\text { nn } \\
\text { ’ } \\
\text { m } \\
= \\
=\end{array}$ & 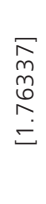 & $\begin{array}{l}\bar{\sigma} \\
\omega \\
m \\
\\
\vdots\end{array}$ & 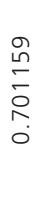 & 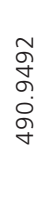 & 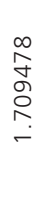 & $\begin{array}{l}\frac{m}{n} \\
\underline{\omega} \\
\stackrel{n}{\sim} \\
\bar{\sim}\end{array}$ & 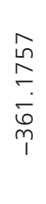 & 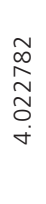 & 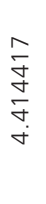 & \begin{tabular}{l}
0 \\
\multirow{1}{*}{} \\
$\infty$ \\
\multirow{1}{*}{} \\
0 \\
$\dot{0}$
\end{tabular} & 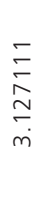 & 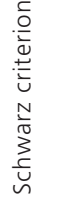 & \\
\hline $\begin{array}{l}\stackrel{\infty}{+} \\
\stackrel{m}{m} \\
\tilde{\sigma} \\
\stackrel{0}{0}\end{array}$ & 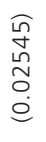 & 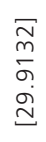 & 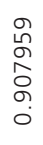 & $\begin{array}{l}0 \\
\circ \\
\circ \\
\text { ஸू } \\
\infty \\
0 \\
0\end{array}$ & $\begin{array}{l}\text { o } \\
+ \\
+ \\
\uplus \\
\stackrel{+}{-} \\
-\end{array}$ & 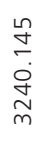 & 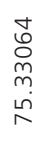 & $\begin{array}{l}\infty \\
\infty \\
0 \\
0 \\
\sim \\
0 \\
\infty \\
1\end{array}$ & $\begin{array}{l}\frac{1}{N} \\
\frac{\sigma}{\sigma} \\
\frac{1}{-}\end{array}$ & 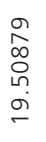 & 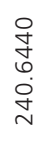 & 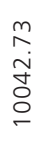 & $\begin{array}{l}\underset{0}{0} \\
0 \\
\stackrel{\sim}{0} \\
\underset{\sim}{\sim}\end{array}$ & 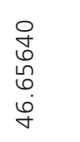 \\
\hline $\begin{array}{l}\stackrel{\text { م }}{\circ} \\
\stackrel{\dot{\sim}}{\Psi} \\
\stackrel{\sim}{+}\end{array}$ & $\begin{array}{l}\widehat{0} \\
0 \\
\dot{u} \\
\emptyset \\
o \\
0\end{array}$ & $\begin{array}{l}\sigma \\
\text { ন } \\
\text { ঠे } \\
\dot{ \pm} \\
\dot{ \pm}\end{array}$ & 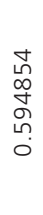 & 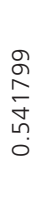 & $\begin{array}{l}\stackrel{n}{\sim} \\
\text { J } \\
\stackrel{+}{~} \\
\stackrel{n}{N}\end{array}$ & $\begin{array}{l}\underset{N}{N} \\
\underset{\sim}{N} \\
-\end{array}$ & 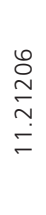 & \begin{tabular}{l}
$\stackrel{+}{+}$ \\
$\infty$ \\
$\circ$ \\
\multirow{1}{*}{} \\
$\stackrel{1}{ }$
\end{tabular} & $\begin{array}{l}\stackrel{n}{0} \\
\mathscr{\theta} \\
\varrho \\
\stackrel{n}{n} \\
m \\
m\end{array}$ & $\begin{array}{l}\stackrel{\bigcirc}{\circ} \\
m \\
m \\
\stackrel{N}{N} \\
\dot{m}\end{array}$ & $\begin{array}{l}\frac{0}{0} \\
\infty \\
\frac{1}{N} \\
\vdots \\
0\end{array}$ & 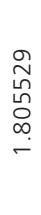 & 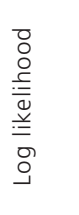 & 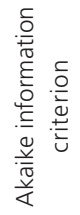 \\
\hline 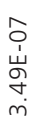 & 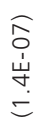 & $\begin{array}{l}\infty \\
\stackrel{\infty}{\sim} \\
\stackrel{\sim}{\sim} \\
\stackrel{\sim}{\simeq}\end{array}$ & 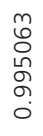 & 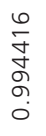 & 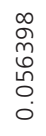 & $\begin{array}{l}\underset{N}{N} \\
\infty \\
\infty \\
\vdots \\
0\end{array}$ & 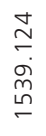 & $\begin{array}{l}m \\
\infty \\
0 \\
0 \\
i n \\
\circ \\
\circ\end{array}$ & 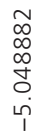 & 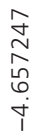 & 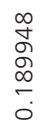 & 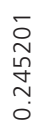 & 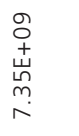 & $\begin{array}{l}\stackrel{\circ}{0} \\
+ \\
\dot{+} \\
\ddot{0} \\
\dot{\sim}\end{array}$ \\
\hline 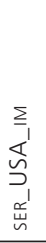 & & & $\begin{array}{l}\frac{\pi}{0} \\
\frac{\pi}{0} \\
\frac{0}{0} \\
\tilde{J} \\
\widetilde{J} \\
\propto\end{array}$ & 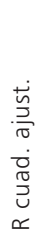 & 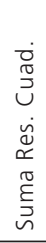 & 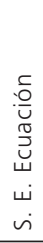 & 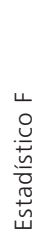 & 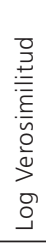 & 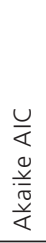 & 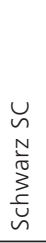 & $\begin{array}{l}\frac{0}{0} \\
\frac{0}{0} \\
\frac{0}{0} \\
\frac{0}{0} \\
\frac{\pi}{0} \\
\frac{0}{2} \\
\Sigma\end{array}$ & 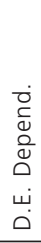 & 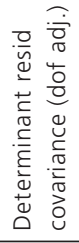 & 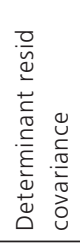 \\
\hline
\end{tabular}


Tabla 12. Prueba de normalidad conjunta para los residuales del grupo de variables elegida mediante criterios de sincronización

\begin{tabular}{cc|ccc}
\hline \multicolumn{4}{c}{ Prueba de normalidad multivariada para residuales de modelo de cointegración } \\
\hline H0: Los residuales son normales multivariados & Ortogonalización: Cholesky (Lutkepohl) \\
\hline Muestra: 1999M01 2015M02 & \multicolumn{3}{|c}{ Observaciones incluidas: 191} \\
\hline Componente & Sesgo & Chi-cuadrada & G. de L. & Probabilidad \\
\hline 1 & -0.164625 & 0.862728 & 1 & 0.3530 \\
2 & -0.353823 & 3.985230 & 1 & 0.0459 \\
3 & 0.354068 & 3.990760 & 1 & 0.0458 \\
4 & -0.020474 & 0.013344 & 1 & 0.9080 \\
5 & 0.236073 & 1.774080 & 1 & 0.1829 \\
6 & -0.010557 & 0.003548 & 1 & 0.9525 \\
7 & 0.018742 & 0.011182 & 1 & 0.9158 \\
8 & 0.181496 & 1.048620 & 1 & 0.3058 \\
\hline
\end{tabular}

Fuente: Elaboracion propia mdiante E-Views 9.

En este caso, se tiene un posible caso de relación espuria (Bondad de ajuste demasiado alta), aunque se cumplen con las pruebas de normalidad de residuales y buena parte de los estimadores son significativos. Este hecho queda abierto para futura investigación.

\section{Conclusiones}

La Industria Manufacturera de Estados Unidos EUA-IM es la serie maestra del sistema, ya que marca los principales cambios en los ciclos de la economía de Canadá y Estados Unidos. Se realizó el análisis con distintas variables de Canadá, pero las Exportaciones totales C-X y las Exportaciones de bienes de Consumo C-XC fueron las que más se sincronizaron con el sistema de Estados Unidos y México.

La variable que marca los principales cambios en México es la Industria Manufacturera M-IM y la Actividad Industrial M-AI, marcando los cambios en el consumo y por lo tanto en el resto de las variables macroeconómicas. Respecto a la Inversión Fija Bruta de México M-IFB, tiene cortes en los ciclos marcados por 
EUA-IM y por M-IM, esta evidencia es importante, ya que puede marcar el ritmo de crecimiento de la economía.

Una vez que se encontró evidencia de la sincronización de los ciclos, se realizó el cálculo de la fase para determinar el tiempo de sincronización de las variables, mostrándose que existe sincronización débil, fuerte y aceleramiento de los ciclos debido al aumento del intercambio comercial.

El Consumo Privado de México M-c y M-IFB se encuentra sincronizado en fase con las variables EUA-IM y con EUA-M (importaciones), teniendo una sincronización fuerte, ya que se presentó antes y después del periodo de crisis. Es importante destacar que algunas variables después de la crisis no pudieron recuperar la sincronización debido a la aceleración de los ciclos, ejemplo de ello es M-AI o M-IFB.

Con las variables que se encontró evidencia de sincronización se realizó el análisis econométrico, el cual sustenta la evidencia encontrada.

\section{REFERENCIAS BIBLIOGRÁFICAS}

Arthur, W. Brian (2013), Complexity Economics: A Different Framework for Economic Thought, Santa Fe, Nuevo Mexico, Complexity Economics Oxford University Press (forthcoming).

Calderón, Cuauhtémoc (2012), "Crisis y ciclos económicos de México de 1896 al 2010", Argumentos, XXV aniversario, núm. 70, septiembre-diciembre, pp. 105-128.

Chang, Tsangyao, Simo-Kengne, Beatrice Desiree y Gupta, Rangan (2013), "The Causal Relationship Between Exports and Economic Growth in the Nine Provinces of South Africa: Evidence from Panel-Granger Causality Test", International Journal of Economic Policy in Emerging Economies, 6 (3), pp. 296-310.

Chian, Abraham C., Rempel, Erico L. y Rogers, Colin (2006), "Complex Economic Dynamics: Chaotic Saddle, Crisis and Intermittency", Chaos, Solitons \& Fractals, 29 (5), pp. 1194-1218.

Chow, Peter C. (1987), Causality Between Export Growth and Industrial Development: Empirial Evidence from the NICs", Journal of Development Economics, 26 (1), pp. 55-63.

Delajara, Marcelo (2012), "Sincronización de ciclos económicos México-Estados Unidos. Nuevos resultados con base en el análisis de los índices coincidentes regionales de México", Documento de Investigación del Banco de México.

Hafner, Christian M. y Manner, Hans (2012), "Dynamic Stochastic Copula Models: Estimation, Inference and Applications", Journal of Applied Econometrics, 27 (2), pp. 269-295. 
Holling, Crawford S. (2001), "Understanding the Complexity of Economic, Ecological, and Social Systems", Ecosystems, 4 (5), pp. 390-405.

Hsueh, Shun-Jen, Hu, Yu-Hau y Tu, Chien-Heng (2013), “Economic Growth and Financial Development in Asian Countries: a Bootstrap Panel Granger Causality Analysis", Economic Modelling, 32, pp. 294-301.

Jung, Woo S. y Marshall, Peyton J. (1985) "Exports, Growth and Causality in Developing Countries", Journal of Development Economics, 18 (1), pp. 1-12.

Kar, Muhsin, Nazlığlu, Şaban. y Ağr, Hüseyin (2011), "Financial Development and Economic Growth Nexus in the Mena Countries: Bootstrap Panel Granger Causality Analysis", Economic Modelling, 28 (1), pp. 685-693.

Kalecki, Michal (1977), Ensayos escogidos sobre dinámica de la economía capitalista, 1933-1970, México, Fondo de Cultura Económica.

Keynes, John Maynard (2005), Théorie générale de l'emploi, de l'intérêt et de la monnaie, París, Payot \& Rivages.

Kitchin, Joseph (1923), "Cycles and Trends in Economic Factors", Review of Economics and Statistics, 5 (1), pp. 10-16.

Marx, Karl (1968), Euvres économie I et II, París, Gallimard.

Mejía Reyes Pablo, Gutierrez Alva E. E y Farias Silva, Claudia A. (2006), "La sincronización de los ciclos económicos de México y Estados Unidos”, Investigación Económica, vol. LXV, num. 258, octubre diciembre, pp.15-45.

Nazlioglu, Saban (2011), "World Oil and Agricultural Commodity Prices: Evidence from Nonlinear Causality”, Energy Policy, 39 (5), pp. 2935-2943.

Pikovsky, A., Rosemblum M. y Kurths J. (2001), Un concepto universal en las ciencias no lineales, Cambridge, Cambridge University Press.

Rodríguez Benavides, Domingo, Lima Santiago, Vicente y Ortiz Edgar (2015), “Sincronizaron México y Estados Unidos, sus ciclos económicos con el TLCAN?", Contaduría y Administración, 60 (S1), pp. 195-229.

Schumpeter, Joseph Alois (1935), Théorie de l'évolution économique, París, Librairie Dalloz.

Sismondi, Jean Charles Leonerde Simonde de (1827), Nouveaux principes de l'Economie Politique, ou de la Richesse, París, Delaunay.

Smith, Michael S., Gan, Quan y Kohn, Robert J. (2012), “Modelling Dependence Using Skew to copulas: Bayesian Inference and Applications", Journal of Applied Econometrics, 27 (3), pp. 500-522.

Sugihara, George, May, Robert, Ye, Hao, Hsieh, Chih-hao, Deyle, Ethan, Fogarty, Michael y Munch, Stephan (2012), "Detecting Causality in Complex Ecosystems”, Science, 338 (6106), pp. 496-500. 
Toda, Hiro Y. y Yamamoto, Taku (1995), "Statistical Inference in Vector Autoregressions with Possibly Integrated Processes", Journal of Econometrics, 66 (1), pp. 225-250.

Tekin, Rifat Baris (2012), "Development Aid, Openness to Trade and Economic Growth in Least Developed Countries: Bootstrap Panel Granger Causality Analysis", Procedia-Social and Behavioral Sciences, 62, pp. 716-721.

Thornton, John (1996), "Cointegration, Causality and Export-Led Growth in Mexico, 1895-1992”, Economics Letters, 50 (3), pp. 413-416.

Volos, Christos K., Kyprianidis, Ioannis M. y Stouboulos, Ioannis N. (2012), “Synchronization Phenomena in Coupled Nonlinear Systems Applied in Economic Cycles", wSEAS Transactions on Systems, 11 (12), pp. 681-690.

Wang, Yudong y Wu, Chongfeng (2012), "Energy Prices and Exchange Rates of the US Dollar: Further Evidence from Linear and Nonlinear Causality Analysis", Economic Modelling, 29 (6). 